\title{
U.S. Hydropower Resource Assessment for Arizona
}

\author{
Prepared by: \\ Alison M. Conner \\ James E. Francfort \\ Project Manager: \\ Ben N. Rinehart
}

Published October 1997

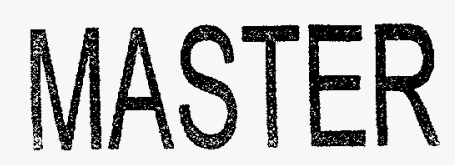

Idaho National Engineering and Environmental Laboratory Renewable Energy Products Department Lockheed Martin Idaho Technologies Company Idaho Falls, Idaho 83415

\author{
Prepared for the \\ U.S. Department of Energy \\ Assistant Secretary for Energy Efficiency and Renewable Energy \\ Under DOE Idaho Operations Office \\ Contract DE-AC07-94ID13223
}




\section{DESCLAMERR}

Portions of this docoment may be Illegible in electronic image produets. Images are produced from the best avaitable original document 


\begin{abstract}
The U.S. Department of Energy is developing an estimate of the undeveloped hydropower potential in the United States. The Hydropower Evaluation Software (HES) is a computer model that was developed by the Idaho National Engineering Laboratory ${ }^{\mathrm{a}}$ for this purpose. HES measures the undeveloped hydropower resources available in the United States, using uniform criteria for measurement. The software was developed and tested using hydropower information and data provided by the Southwestern Power Administration. It is a menu-driven program that allows the personal computer user to assign environmental attributes to potential hydropower sites, calculate development suitability factors for each site based on the environmental attributes present, and generate reports based on these suitability factors. This report describes the resource assessment results for the State of Arizona.
\end{abstract}

a. In January 1997, the name of the Idaho National Engineering Laboratory (INEL) was changed to the Idaho National Engineering and Environmental Laboratory (INEEL). INEEL will be used throughout the text of the document, except where the use of INEL is historically important. 


\section{ACKNOWLEDGMENTS}

The authors thank Peggy A. M. Brookshier and John V. Flynn of the U.S. Department of Energy for their active participation and timely comments. 


\section{CONTENTS}

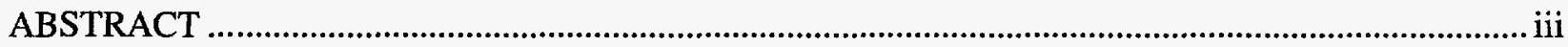

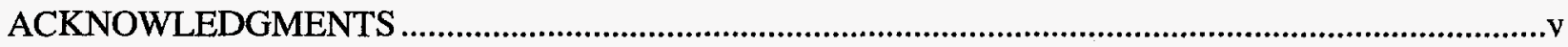

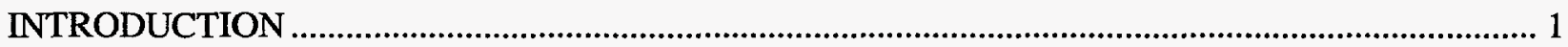

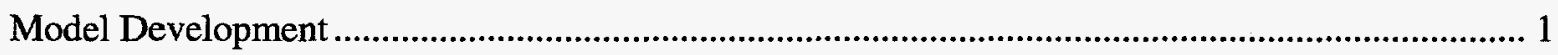

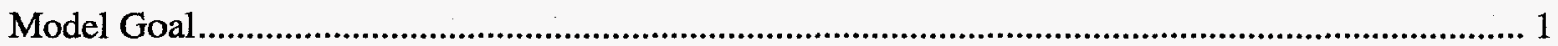

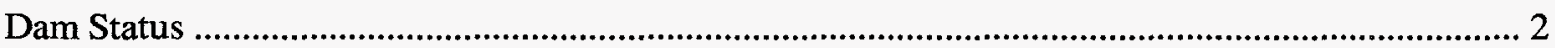

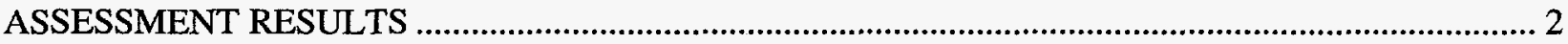

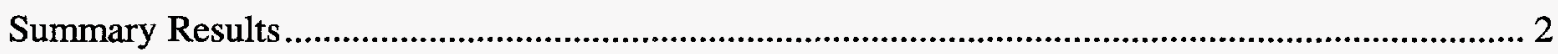

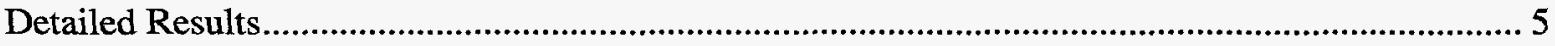

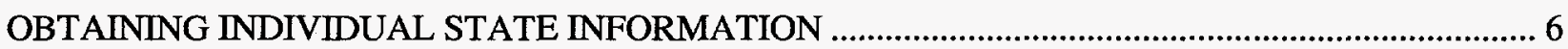

ADDITIONAL HYDROPOWER EVALUATION SOFTWARE INFORMATION ............................... 6

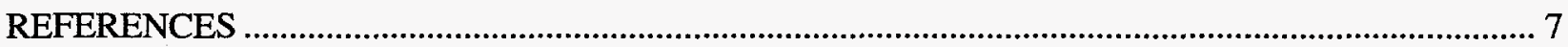

Appendix A-Summary Report

Appendix B-River Basins Report

Appendix C-Arizona Sites List

Appendix D-Individual Resource Database List

\section{FIGURES}

1. Number of sites, by capacity groups, with HES-modeled undeveloped hydropower potential........... 3

2. The nonmodeled and HES-modeled undeveloped hydropower potential ........................................ 3

3. The number of sites with undeveloped hydropower potential and the total megawatts of HES-modeled undeveloped hydropower potential. 
4. Number of sites with undeveloped hydropower potential in the Arizona river basins...

5. Megawatts of HES-modeled undeveloped hydropower potential in the Arizona river basins 5

\section{TABLES}

1. Undeveloped hydropower potential summary for Arizona ................................................... 2 


\section{U.S. Hydropower Resource Assessment for Arizona}

\section{INTRODUCTION}

In June 1989, the U.S. Department of Energy initiated the development of a National Energy Strategy to identify the energy resources available to support the expanding demand for energy in the United States. Public hearings conducted as part of the strategy development process indicated that undeveloped hydropower resources were not well defined. As a result, the Department of Energy established an interagency Hydropower Resource Assessment Team to ascertain the undeveloped hydropower potential. In connection with these efforts by the Department of Energy, the Idaho National Engineering Laboratory designed the Hydropower Evaluation Software (HES), which has been used to perform a resource assessment of the undeveloped conventional hydropower potential in over 30 states. This report presents the results of the hydropower resource assessment for the State of Arizona. Undeveloped pumped storage hydropower potential is not included.

The HES was developed as a tool to measure undeveloped hydropower potential regionally or by state. The software is not intended to provide precise development factors for individual sites, but to provide regional or state totals. Because the software was developed as a generic measurement tool encompassing national issues, regional and state totals must be considered judiciously; various local issues may skew undeveloped hydropower potential totals. The information for the resource assessment was compiled from the Federal Energy Regulatory Commission's Hydroelectric Power Resources Assessment database and several other sources. Refer to DOE/ID-10338, the User's Manual (Francfort, Matthews, Rinehart 1991) for the specifics of the software and to DOE/DD-10430.1, the Status Report (Conner, Francfort, Rinehart 1996) for an overview of all resource assessment activities to date.

\section{Model Development}

Hydropower Evaluation Software, both a probability-factor computer model and a database, is a menu-driven program that is intended to be user-friendly. Computer screens and report-generation capabilities were developed to meet the needs of users nationwide. The software uses environmental attribute data to generate an overall project environmental suitability factor (PESF) between 0.1 and 0.9 , where 0.9 indicates the highest likelihood of development and 0.1 indicates the lowest likelihood of development. The suitability factors depend on the unique environmental attributes of each potential site. They reflect the considerations that (a) environmental concerns can make a potential site unacceptable, prohibiting its development (for a suitability factor of 0.1 , or (b) if there are no environmental concerns, there is no negative effect on the likelihood of site development (for a suitability factor of 0.9). A combination of attributes can result in a lower suitability factor because multiple environmental considerations would reduce the likelihood that a site may be developed to its physical potential.

\section{Model Goal}

The goal of the HES is to assemble an accurate resource database of all sites with undeveloped hydropower potential in the United States for use as a planning tool to determine the viable national hydropower potential. Undeveloped hydropower potential is not limited to the development of new sites; it also includes the development of additional hydropowergenerating capacity at sites that currently have hydropower, but are not developed to their full potential. This undeveloped hydropower potential is a source of nonpolluting, renewable energy available to meet the growing power needs of the United States. The HES should help make this goal obtainable and ensure a set of uniform criteria for national assessment. 


\section{Dam Status}

The effects of environmental attributes vary by dam status. The dam status classifications used are as follows:

$$
\begin{gathered}
\text { W = Developed hydropower site } \\
\text { with current power generation, } \\
\text { but the total hydropower po- } \\
\text { tential has not been fully de- } \\
\text { veloped. Only the undeveloped } \\
\text { hydropower potential is dis- } \\
\text { cussed in this report. }
\end{gathered}
$$

$\mathrm{W} / \mathrm{O}=$ Developed site without current power generation. The site has some type of developed impoundment or diversion structure, but no developed hydropower generating capability.

$\mathrm{U}=$ Undeveloped site. The site does not have power generation capability nor a developed impoundment or diversion structure.

\section{ASSESSMENT RESULTS Summary Results}

A total of 21 sites (Table 1) have been identified and assessed for their undeveloped hydropower potential. The HES results for individual site capacities range from 113 kilowatts (kW) to 137 megawatts (MW). The majority, or $86 \%$, of the HES-modeled sites in Arizona are less than $10 \mathrm{MW}$ each (Figure 1).

The nonmodeled undeveloped hydropower potential total for Arizona was identified as 1,809 MW. The HES results lowers this estimate about $81 \%$ to $339 \mathrm{MW}$. The greatest reduction in undeveloped hydropower potential, by MW, occurs at sites with no current power generation capability nor impoundment or diversion structure in place (undeveloped category [U]). These sites have an HESmodeled undeveloped hydropower potential of $166 \mathrm{MW}$, an $89 \%$ or $1,386-\mathrm{MW}$ reduction in the estimated undeveloped hydropower potential (Figure 2). Figure 3 correlates the number of sites that have undeveloped hydropower potential with the total megawatts of HES-modeled undeveloped hydropower potential. The developed sites with power have the largest average undeveloped hydropower potential of $79 \mathrm{MW}$ per site (Figure 3).

The 21 identified sites are located within two major river basins, the Colorado Main Stream River Basin and the Gila River Basin. There are two sites located within the Colorado Main Stream River Basin and 19 sites within the Gila River Basin (Figure 4). Although the Colorado Main Stream River Basin only contains 2 of the 21 sites, it has more undeveloped hydropower potential than the Gila River Basin (Figure 5). It should be noted that $76 \%$ of the HES-modeled undeveloped hydropower potential in the State of Arizona is contained within two sites, AZ007 and AZ008.

Table 1. Undeveloped hydropower potential summary for Arizona. The table contains the nonmodeled undeveloped nameplate potential and the HES-modeled undeveloped potential totals.

\begin{tabular}{lccc}
\hline & Number of projects & Nameplate potential & HES-modeled potential \\
& & $(\mathrm{MW})$ & $(\mathrm{MW})$ \\
\cline { 2 - 4 } With Power & 2 & 206.8 & 157.4 \\
W/O Power & 6 & 50.5 & 15.4 \\
Undeveloped & 13 & $1,552.1$ & 166.3 \\
State Total & 21 & $1,809.4$ & 339.1 \\
\hline
\end{tabular}




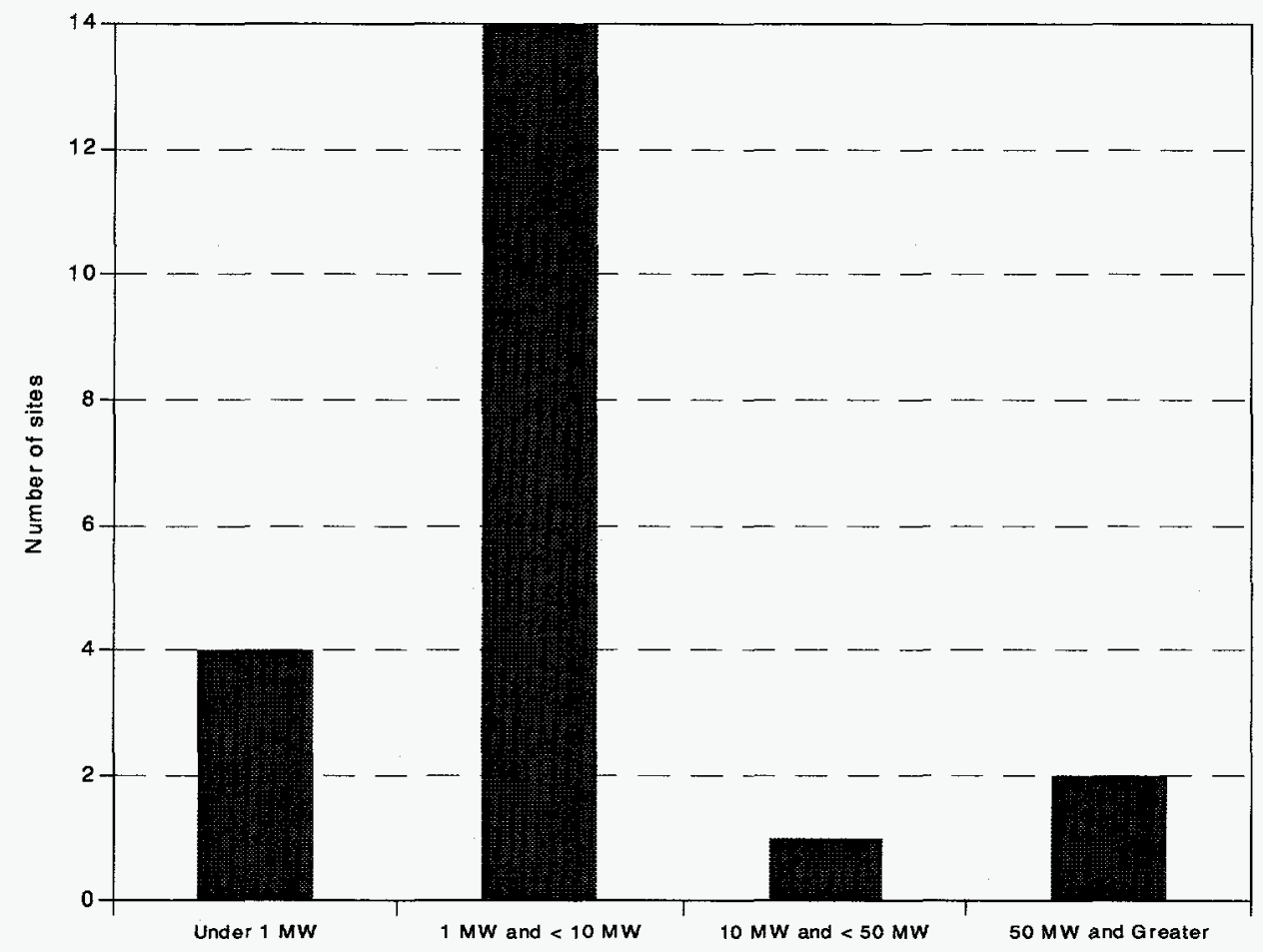

Figure 1. Number of sites, by capacity groups, with HES-modeled undeveloped hydropower potential.

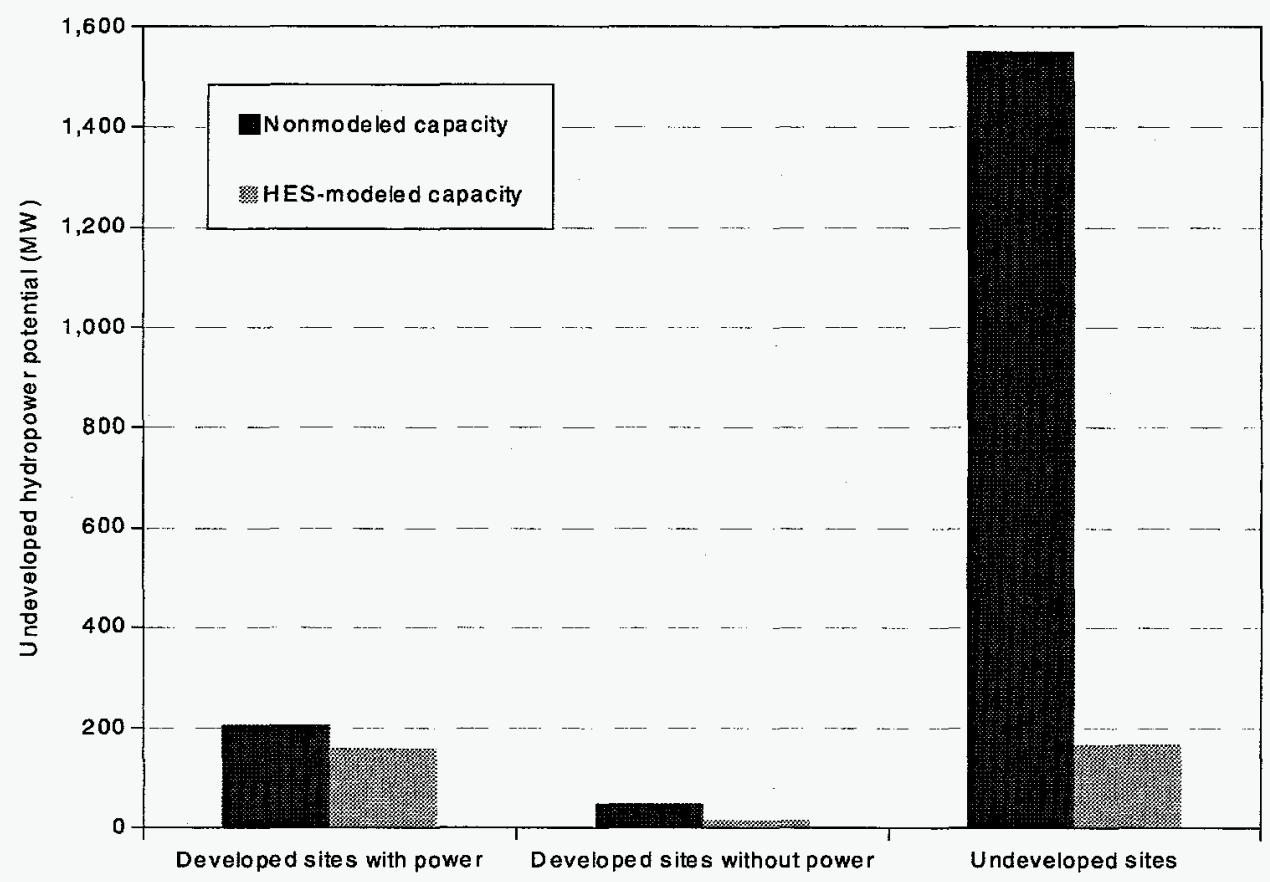

Figure 2. The nonmodeled and HES-modeled undeveloped hydropower potential. 


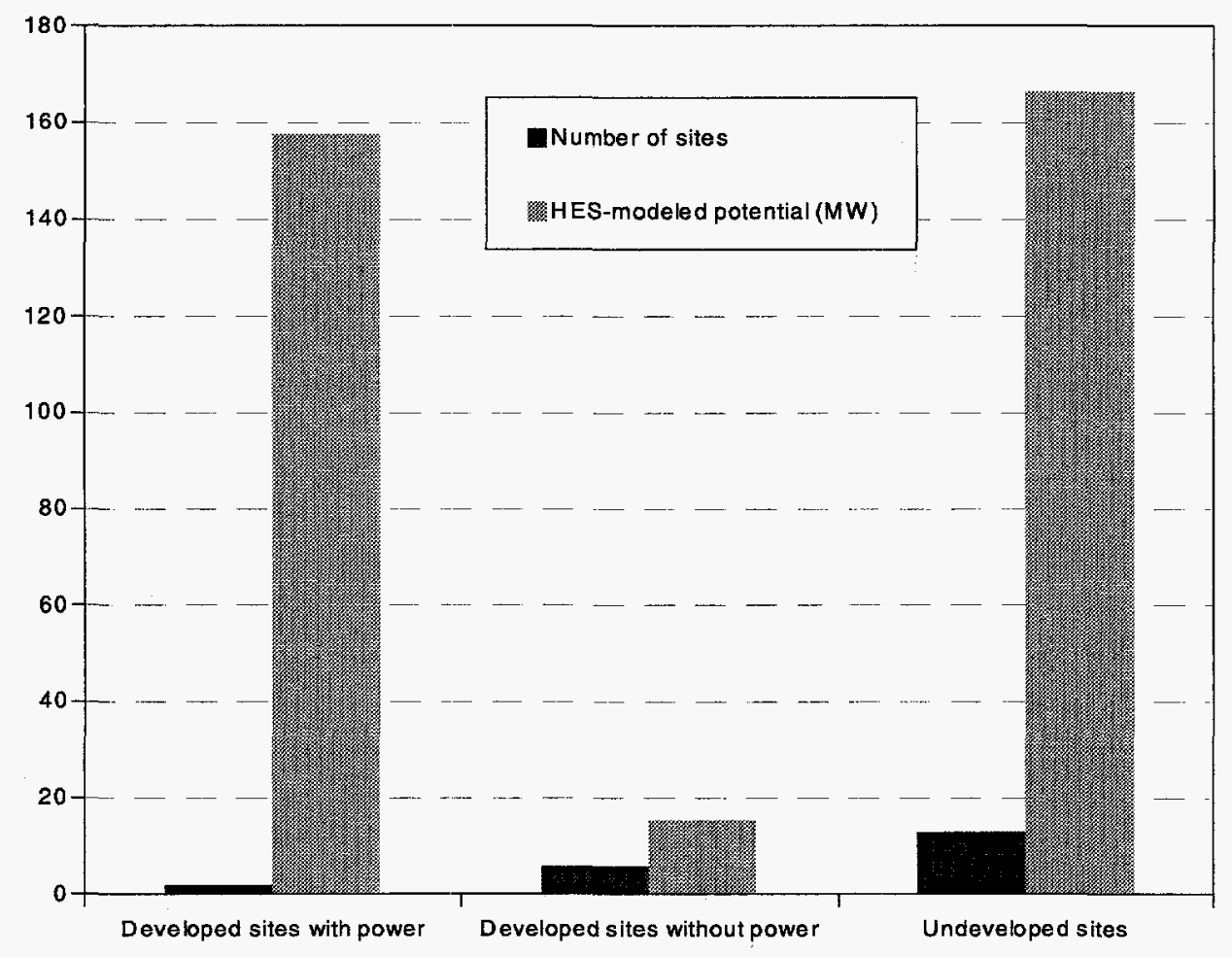

Figure 3. The number of sites with undeveloped hydropower potential and the total megawatts of HES-modeled undeveloped hydropower potential.

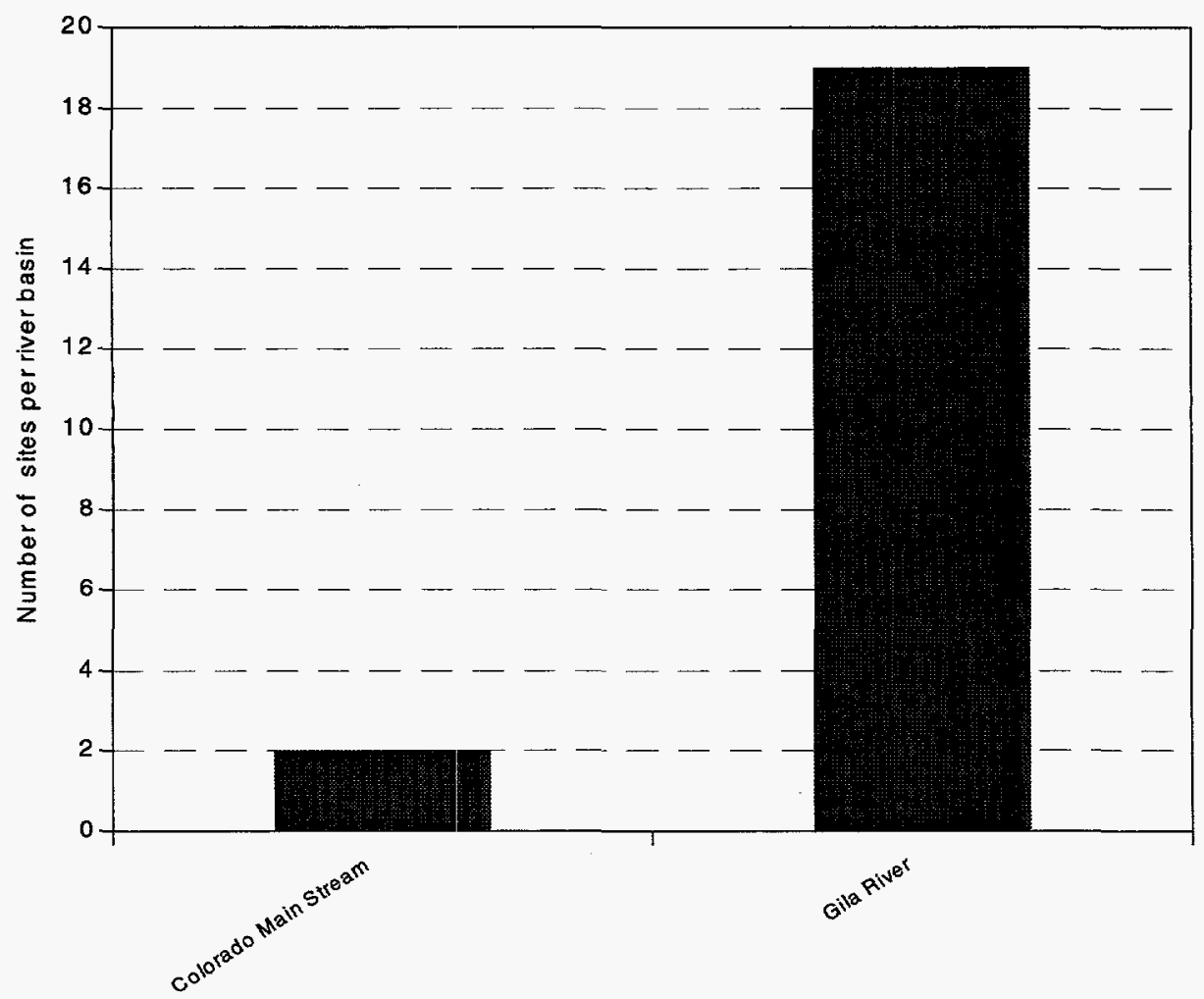

Figure 4. Number of sites with undeveloped hydropower potential in the Arizona river basins. 


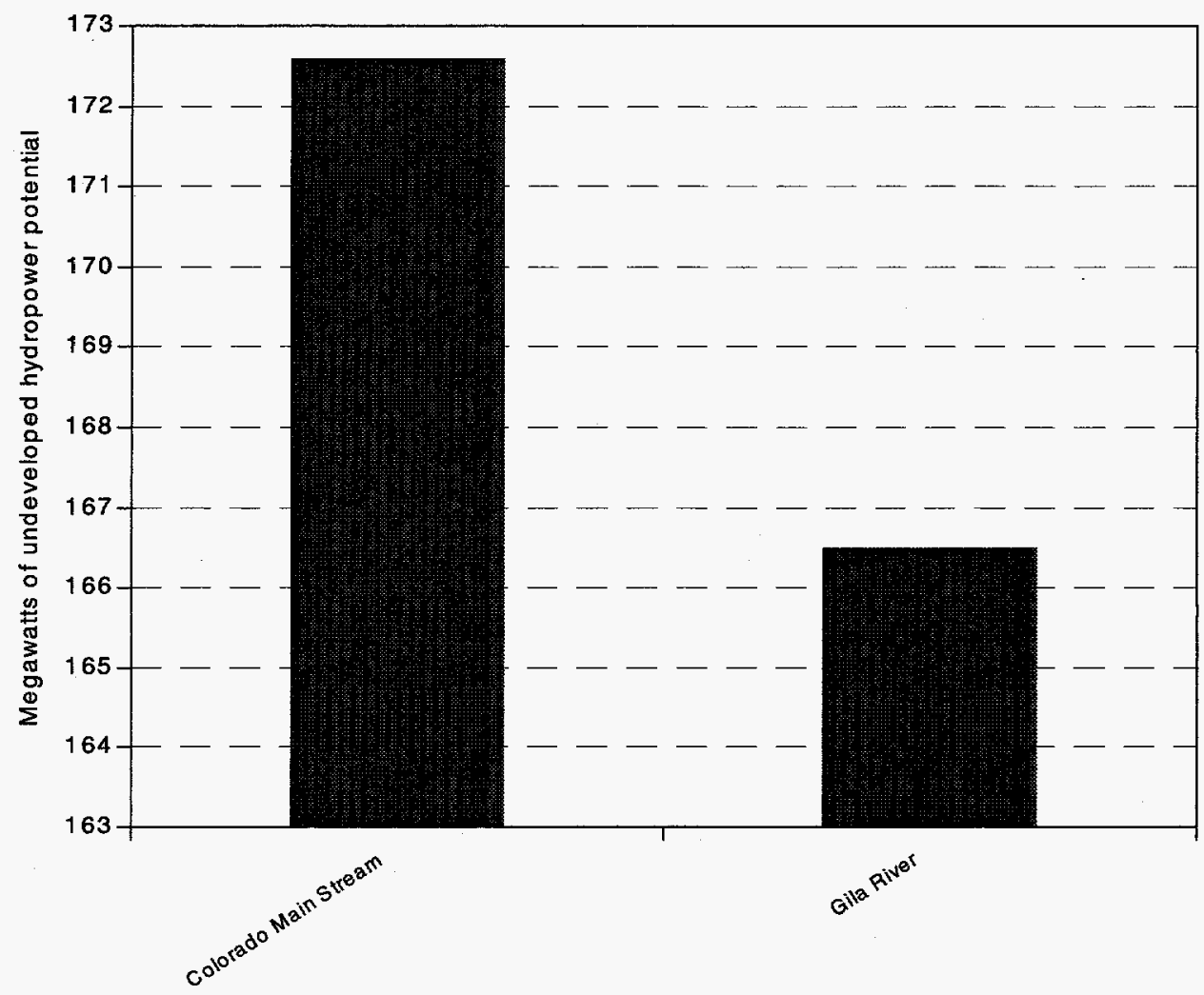

Figure 5. Megawatts of HES-modeled undeveloped hydropower potential in the Arizona river basins.

\section{Detailed Results}

The appendices contain, in the form of HES-generated reports, detailed information about the undeveloped hydropower potential in Arizona. The appendices contain the following information:

Appendix A summarizes the undeveloped hydropower potential by dam status groups. The number of sites, nonmodeled undeveloped hydropower potential, and HES-modeled undeveloped hydropower potential is provided based on the dam status.

Appendix B provides the hydropower resource assessment by river basin, which includes the project number, project name, stream name, dam status, nonmodeled undeveloped hydropower potential, and the HES-modeled undeveloped hydropower potential for each site. Subtotals are provided for each river basin.
Appendix $\mathbf{C}$ lists the project numbers, plant name, stream name, if a site is Federally owned, nonmodeled undeveloped hydropower potential, and HES-modeled undeveloped hydropower potential. The sites are grouped by dam status.

Appendix D contains a resource database list for the 21 sites in Arizona. Information includes plant name, stream, state, county, river basin and owner names, project number, nameplate and HES-modeled undeveloped hydropower potential, the unit and plant types, dam status, latitude, longitude, and the environmental factors that the HES uses to determine the PESF. 


\section{OBTAINING INDIVIDUAL STATE INFORMATION}

Additional copies of the hydropower resource assessment results for individual states are available and can be obtained by writing or calling the authors or the National Technical Information Service (NTIS).

Telephone Orders-(703) 487-4650. NTIS sales desk and customer services are available between 8:30 a.m. and 5:00 p.m., Eastern Standard Time.

Fax-(703) 321-8547. Customers may fax their orders to NTIS. These orders may be charged to a NTIS deposit account, American Express, VISA, or MasterCard.

Mail Orders-Mail orders should be sent to National Technical Information Service, Document Sales, 5285 Port Royal Road, Springfield, VA 22161. Call the sales desk for prices before placing an order.

E-mail-Customers may e-mail their requests to info@ntis.fedworld.gov.

Method of Payment-Customers may pay for reports (and other NTIS products and services) by (a) credit card (American Express, Visa or MasterCard); (b) check or money order on a United States bank payable to NTIS; (c) NTIS deposit account; or, (d) by asking to be billed (add $\$ 7.50$ per order), United States, Canada, and Mexico, only.

Handling Fee $-\mathrm{A} \$ 4.00$ handling fee per total order applies to orders from the United States, Canada, and Mexico. Handling charges do not apply to rush order service or pick-up orders.

Postage and Shipping-Orders are shipped first class mail, or equivalent, to addresses in the United States, Canada, and Mexico.
Order Turnaround Time-Orders for technical reports generally are shipped within 3 to 5 days of receipt. For faster service, NTIS offers rush order service.

Rush Order Service-Call 1-800-533-NTIS. In Virginia, Canada, and Mexico call (703) $487-4700$. For NTIS rush order service add $\$ 15$ per item. This guarantees that an order will be processed through NTIS within 24 hours of its receipt. These orders receive immediate, individual attention. The items ordered are delivered by first call mail. Call NTIS for information on rush order service for computer products.

For Help in Tracing an Order-Call (703) 487-4650 and request the customer service option.

\section{ADDITIONAL HYDROPOWER EVALUATION SOFTWARE INFORMATION}

Additional information concerning the HES can be obtained by contacting Ben Rinehart or Jim Francfort at the addresses provided below. Copies of the software and the User's Manual may also be obtained from these individuals.

Ben Rinehart, Project Manager

Idaho National Engineering and Environmental Laboratory

P.O. Box 1625, MS 3830

Idaho Falls, ID 83415-3830

(208) 526-1002

Jim Francfort

Idaho National Engineering and Environmental Laboratory

P.O. Box 1625, MS 3830

Idaho Falls, ID 83415-3830

(208) 526-6787 


\section{REFERENCES}

Conner, A. M., J. E. Francfort, and B. N. Rinehart, 1996, Uniform Criteria for U.S. Hydropower Resource Assessment, Hydropower Evaluation Software Status Report-II, DOE/ID 10430.1, Idaho National Engineering Laboratory, Idaho Falls, Idaho.

Francfort, J. E., S. D. Matthews, and B. N. Rinehart, 1991, Hydropower Evaluation Software User's Manual, DOE/ID-10338, Idaho National Engineering Laboratory, Idaho Falls, Idaho. 
Appendix A

Summary Report 
DATE: $08 / 26 / 97$

$H Y D R O P O W E R$

Number of

state

Category

Projects

With Power

W/O Power

Undeveloped

STATE TOTAL

TOTALS

With Power

W/O Power

Undeveloped

GRAND TOTAI
C A P A C I T Y

\section{Name Plate} Capacity (KW)

206816

50500

1552056

1809372

206816

50500

1552056

1809372
HES Adjusted Capacity (KW)
157408

15355

166290.40

339053.40

157408

15355

166290.40

339053.40 


\section{Appendix B}

River Basins Report 


\begin{tabular}{|c|c|c|c|c|c|}
\hline & HYDROPOWER RESOURCE & ASSESSM & ENT BY RIVER & BASIN & \\
\hline $\begin{array}{c}\text { FERC } \\
\text { Number }\end{array}$ & $\begin{array}{c}\text { Plant Name/ } \\
\text { Stream }\end{array}$ & $\begin{array}{l}\text { Dam } \\
\text { stat }\end{array}$ & $\begin{array}{l}\text { Name Plate } \\
\text { Rating (KW) }\end{array}$ & PESF & PESF * KW \\
\hline $\begin{array}{l}* * \text { Rive } \\
\text { AZ008 }\end{array}$ & $\begin{array}{l}x \text { Basin COLORADO MAIN STREAM } \\
\text { HUALAPAI } \\
\text { COLORADO R }\end{array}$ & U & 1366000.00 & 0.10 & 136600.00 \\
\hline AZ005 & $\begin{array}{l}\text { GLEN CANYON } \\
\text { COLORADO } R\end{array}$ & W & 71816.00 & 0.50 & 35908.00 \\
\hline ** Subt & otal ** & & 1437816.00 & & 172508.00 \\
\hline $\begin{array}{l}* * \text { Rive } \\
\text { AZ015 }\end{array}$ & $\begin{array}{l}\text { Basin GILA RIVER BASIN } \\
\text { YUMA MAIN CANAL } \\
\text { YUMA MN CNL (GILA R) }\end{array}$ & $\mathrm{U}$ & 1410.00 & 0.90 & 1269.00 \\
\hline $\mathrm{AZOO} 2$ & $\begin{array}{l}\text { BEARDSIEY CANAI DROP } \\
\text { BEARDSIEY CNL (AQUA FRIA R) }\end{array}$ & $\mathrm{U}$ & 1000.00 & 0.90 & 900.00 \\
\hline AZ003 & $\begin{array}{l}\text { CAP CANAL TURNOUT } \\
\text { CAP CNL (AQUA FRIA R) }\end{array}$ & $\mathrm{U}$ & 2500.00 & 0.90 & 2250.00 \\
\hline AZ013 & $\begin{array}{l}\text { WADDELI } \\
\text { AQUA FRIA } R\end{array}$ & $\mathrm{U}$ & 1500.00 & 0.90 & 1350.00 \\
\hline AZ012 & $\begin{array}{l}\text { ORME } 3 \\
\text { ORME CNL (SALT R) }\end{array}$ & $\mathrm{U}$ & 20000.00 & 0.10 & 2000.00 \\
\hline 11057 & $\begin{array}{l}\text { ROOSEVELT (RWCD) } \\
\text { ROOSEVELT CNL (SALT R) }\end{array}$ & U & 3200.00 & 0.90 & 2880.00 \\
\hline AZ010 & $\begin{array}{l}\text { LIVINGSTONE } \\
\text { PINAI CR, SALT R }\end{array}$ & $\mathrm{U}$ & 11500.00 & 0.10 & 1150.00 \\
\hline AZ004 & $\begin{array}{l}\text { GLEASON FLAT } \\
\text { SALT R }\end{array}$ & $\mathrm{U}$ & 43500.00 & 0.10 & 4350.00 \\
\hline AZ011 & $\begin{array}{l}\text { MULE HOOF } \\
\text { SALT R }\end{array}$ & $\mathrm{U}$ & 43500.00 & 0.10 & 4350.00 \\
\hline AZ014 & $\begin{array}{l}\text { WALNUT CANYON } \\
\text { SALT R }\end{array}$ & $\mathrm{U}$ & 25200.00 & 0.10 & 2520.00 \\
\hline AZ009 & $\begin{array}{l}\text { KNOB } \\
\text { SALT R }\end{array}$ & $\mathrm{U}$ & 28500.00 & 0.10 & 2850.00 \\
\hline 11178 & $\begin{array}{l}\text { TUCSON } \\
\text { TUCSON WS PL (CAP AQUADUCT) }\end{array}$ & $\mathrm{U}$ & 4246.00 & 0.90 & 3821.40 \\
\hline AZ007 & $\begin{array}{l}\text { HOOVER } \\
\text { COLORADO } \mathbf{R}\end{array}$ & $\mathbf{W}$ & 135000.00 & 0.90 & 121500.00 \\
\hline
\end{tabular}


Page No.

$08 / 26 / 97$

FERC

Number
2

HYDROPOWER RESOURCE ASSESSMENT BY RIVER BASIN

Plant Name/

stream

\section{Dam Name Plate}

stat Rating (KW) PESF PESF * KW

พั

$150.00 \quad 0.75$

wo

$700.00 \quad 0.90$

wo

wo

wo

wo

$750.00 \quad 0.75$

371556.00

166545.40

1809372.00

112.50

630.00

9700.00

1750.00

2600.00

562.50
07275 NEW WADDELL AGUA FRIA R

AZ001 ARIZONA FALIS ARIZONA CNL

03953 BARTLETT

VERDE R

03736 HORSESHOE

VERDE $R$

AZ006 HEADGATE ROCK COLORADO $R$

10485

CHANDLER FALLS TEMPE CNL (SALT R)
** Subtotal **

*** Total *** 


\section{Appendix C}

\section{Arizona Sites List}


Page No.

$08 / 26 / 97$

1

HYDROPOWER RESOURCE ASSESSMENT BY FERC NUMBER

Plant Name/

Stream

** FERC Number 11057

ROOSEVELT (RWCD)

ROOSEVELT CNL (SALT R)

** Subtotal **

** FERC Number 11178

TUCSON

TUCSON WS PL (CAP AQUADUCT)

** Subtotal **

** FERC Number AZOO2

BEARDSLEY CANAL DROP

BEARDSLEY CNL (AQUA FRIA R)

** Subtotal **

** FERC Number AZ003

CAP CANAL TURNOUT

CAP CNL (AQUA FRIA R)

** Subtotal **

** FERC Number AZ004

GLEASON FLAT

SALT R

** Subtotal **

** FERC Number AZ008

HUALAPAI

COLORADO $\mathbf{R}$

** Subtotal **

** FERC Number AZ009

KNOB

SAIT R
Dam Name Plate

ST stat Rating (KW) PESF

PESF * KW

$\mathrm{AZ} \quad \mathrm{U}$

$3200.00 \quad 0.90$

2880.00

3200.00

2880.00

$A Z \quad U$

$4246.00 \quad 0.90$

3821.40

4246.00

3821.40

AZ U

$1000.00 \quad 0.90$

900.00

1000.00

900.00

AZ U

$2500.00 \quad 0.90$

2250.00

2500.00

2250.00

AZ U

$43500.00 \quad 0.10$

4350.00

43500.00

4350.00

AZ U

1366000.000 .10

136600.00

1366000.00

136600.00

AZ U

$28500.00 \quad 0.10$

2850.00 
HYDROPOWER RESOURCE ASSESSMENT BY FERC NUMBER

Plant Name/

Stream

** Subtotal **

** FERC Number AZ010

LIVINGSTONE

PINAI CR, SALT R

** Subtotal **

** FERC Number AZ011

MULE HOOF

SALT $R$

** Subtotal **

** FERC Number AZ012

ORME 3

ORME CNL(SALT R)

** Subtotal **

** FERC Number AZ013

WADDELI

AQUA FRIA R

** Subtotal **

** FERC Number AZ014

WAINUT CANYON

SAIT $R$

** Subtotal **

** FERC Number AZ015

YUMA MAIN CANAL

YUMA MN CNL (GIIA R)

** Subtotal ** $\begin{array}{lll}\text { Dam } & \text { Name Plate } \\ \text { ST Stat } & \text { Rating (KW) PESF } \quad \text { PESF * KW }\end{array}$

$$
28500.00
$$

2850.00

$\mathrm{AZ} \quad \mathrm{U}$

$11500.00 \quad 0.10$

1150.00

11500.00

1150.00

$\mathrm{AZ} \quad \mathrm{U}$

$43500.00 \quad 0.10$

4350.00

43500.00

4350.00

$\mathrm{AZ} \quad \mathrm{U}$

$20000.00 \quad 0.10$

2000.00

20000.00

2000.00

$A Z U$

$1500.00 \quad 0.90$

1350.00

1500.00

1350.00

$\mathrm{AZ} U$

$25200.00 \quad 0.10$

2520.00

25200.00

2520.00

$A Z \quad U$

$1410.00 \quad 0.90$

1269.00

1410.00

1269.00 
Page No.

$08 / 26 / 97$
3

HYDROPOWER RESOURCE ASSESSMENT BY FERC NUMBER

Plant Name/

Stream

** FERC Number AZ005

GLEN CANYON

COLORADO $\mathbf{R}$

** Subtotal **

** FERC Number AZ007

HOOVER

COLORADO R

** Subtotal **

** FERC Number 03736

HORSESHOE

VERDE $R$

** Subtotal **

** FERC Number 03953

BARTLETT

VERDE $\mathbf{R}$

** Subtotal **

** FERC Number 07275

NEW WADDELL

AGUA FRIA $R$

** Subtotal **

** FERC Number 10485

CHANDLER FALLS

TEMPE CNL (SALT R)

** Subtotal **

** FERC Number AZ001

ARIZONA FALIS

ARIZONA CNL $\begin{array}{lll}\text { Dam Name Plate } & \\ \text { ST Stat Rating (KW) PESF } & \text { PESF * KW }\end{array}$

$A Z$ W

$71816.00 \quad 0.50$

35908.00

71816.00

35908.00

$\mathrm{AZ} W$

$135000.00 \quad 0.90$

121500.00

135000.00

121500.00

AZ Wo

$3500.00 \quad 0.50$

1750.00

3500.00

1750.00

Az wo

$19400.00 \quad 0.50$

9700.00

19400.00

9700.00

Az wo

$150.00 \quad 0.75$

112.50

150.00

112.50

$\mathrm{Az}$ wo

$750.00 \quad 0.75$

562.50

750.00

562.50

Az พ०

$700.00 \quad 0.90$

630.00 
Page No.

08/26/97
4

HYDROPOWER RESOURCE ASSESSMENT BY FERC NUMBER

Plant Name/

Stream

Dam Name Plate

ST Stat Rating (KW) PESF

$$
700.00
$$

630.00

AZ Wo

26000.00

0.10

2600.00

COLORADO $\mathbf{R}$

26000.00

2600.00

1809372.00

339053.40 
Appendix D

Individual Resource Database List 
RES O UR C E D A T A B A S E I I S T I N G

DATE : $08 / 26 / 97$

PAGE NO: 1

FERC

Number

Plant Name

stream

State

Number

HORSESHOE

VERDE R

Name

03736

county Name

River Basin

MARICOPA

GILA RIVER BASIN

Class Owner Name

$R$ MITCHELL ENERGY CO INC

Name Plate

PESF

Annual Energy

Rating ( $\mathrm{KW}$ )

PESF*KW

Rating (MWh)

PESF Annual

3500.00

$0.50 \quad 1750.00$

17000.00

Energy Rating (MWh)

AZ

\begin{tabular}{|c|c|c|c|c|c|}
\hline $\begin{array}{l}\text { Unit } \\
\text { Type }\end{array}$ & $\begin{array}{l}\text { Plant } \\
\text { Type }\end{array}$ & $\begin{array}{l}\text { Project } \\
\text { Status }\end{array}$ & $\begin{array}{l}\text { Dam } \\
\text { Status }\end{array}$ & $\begin{array}{l}\text { Latitude } \\
\text { Longitude }\end{array}$ & $\begin{array}{r}3359.00 \\
11143.00\end{array}$ \\
\hline C & STG & $x X$ & wo & & \\
\hline
\end{tabular}

Factor

Exist Prob

Factor

Exist Prob

Wild/Scenic Protection

wild/Scenic Tributary or

Upstream/Downstream

wild/Scenic Location

Cultural value

Fish Presence value

L

Geologic Value

\subsection{0}

wildlife Value

Threatened/Endangered Fish

8500

Historic Value Threatened/Endangered Wildlif

Exist Prob

other value

Recreation value

Scenic Value

$\begin{array}{cll} & & \text { Threatened/Endangered Wildlife } \\ & 0.75 & \text { Federal Land Code 103 } \\ 0.90 & \text { Federal Land Code } 104 \\ 0.90 & \text { Federal Land Code 105 } \\ 0.90 & \text { Federal Land Code } 106 \\ 0.90 & \text { Federal Land Code } 107 \\ 0.90 & \text { Federal Land Code } 108 \\ 0.90 & \text { Federal Land code } 198\end{array}$

0.90

0.90

0.90

0.90

$Y$

0.75

0.90

0.90

0.90

0.90

0.90

0.90 
R E S O U R C E

DATE: $08 / 26 / 97$
D A T A B A S E

$S$

I I S T N G

PAGE NO: 2
FERC

Number

Plant Name

03953 BARTLETT

county Name

MARICOPA stream

VERDE $R$

River Basin

GIIA RIVER BASIN state

Name

AZ

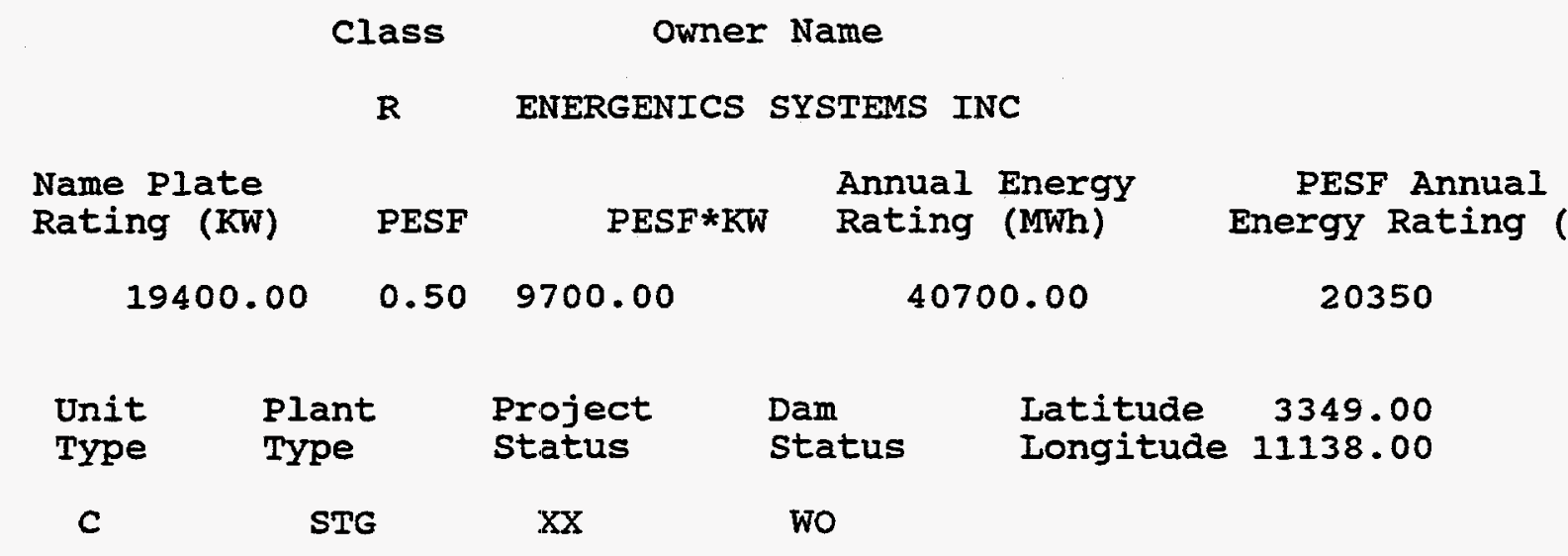

Factor

Exist Prob

Factor

Exist Prob

wild/scenic Protection

wild/scenic Tributary or

Upstream/Downstream wild/Scenic Location

Cultural Value

Fish Presence Value

Geologic Value

Historic Value

other Value

Recreation Value

Scenic Value

Exist Prob

Factor

0.90 Wildife value

Threatened/Endangered Fish

Threatened/Endangered Wildlife

Y 0.75 Federal Land Code 103

0.90 Federal Land Code 104

0.90 Federal Land Code 105

0.90 Federal Land Code 106

0.90 Federal Land Code 107

0.90 Federal Land Code 108

0.90 Federal Land Code 198

0.90

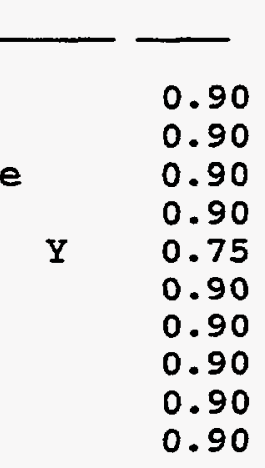


DATE: $08 / 26 / 97$

FERC

Number

07275

NEW WADDELL

County Name

MARICOPA

Class

Owner Name

R GENTRY RESOURCES CORP

Name Plate

Rating (KW)

PESF

Annual Energy

150.00

$0.75 \quad 112.50$

340.00

PAGE NO: 3

stream

State

Name

AZ

River Basin

GIIA RIVER BASIN

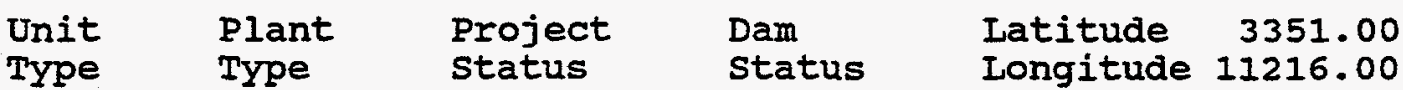

C STG XX wo

Factor

Wild/Scenic Protection

wild/Scenic Tributary or

Upstream/Downstream

wild/Scenic Location

Cultural value

Fish Presence Value

Geologic Value

Historic Value

Other Value

Recreation value

Scenic Value
Exist Prob

0.90

0.90

0.90

0.90

0.90

0.90

0.90

0.90

0.90
PESF Annual Energy Rating (MWh)

255
Factor

Exist Prob

Wildlife Value Threatened/Endangered Fish Threatened/Endangered Wildlife Federal Land Code 103 Federal Iand Code 104 Federal Land Code 105 Federal Land Code 106 Federal Iand Code 107 Federal Land Code 108 Federal Land code 198
0.90

0.90

0.90

0.90

$\mathrm{Y} \quad 0.75$

0.90

0.90

0.90

0.90

0.90 
R E S O U R C E

DATE: $08 / 26 / 97$
D A T A B A S E

PAGE NO: 4

\section{FERC}

Number

10485
Plant Name

CHANDIER FAILS

county Name

MARICOPA stream

TEMPE CNL (SALT R)

River Basin

GILA RIVER BASIN
State

Name

AZ

$\begin{array}{cc}\text { Class } & \text { Owner Name } \\ \text { R } & \text { CHANDLER FALLS HYDRO PNSP }\end{array}$

Name Plate PESF

Annual Energy Rating (KW)

$0.75 \quad 562.50$ Rating (MWh)

PESF Annual Energy Rating (NWh)

$$
750.00
$$

4700.00

3525

\begin{tabular}{|c|c|c|c|}
\hline $\begin{array}{l}\text { Unit } \\
\text { Type }\end{array}$ & $\begin{array}{l}\text { Plant } \\
\text { Type }\end{array}$ & $\begin{array}{l}\text { Project } \\
\text { status }\end{array}$ & $\begin{array}{l}\text { Dam } \\
\text { status }\end{array}$ \\
\hline C & DIV & $X X$ & wo \\
\hline
\end{tabular}

Factor

Exist Prob

Factor

Exist Prob

Wild/scenic Protection

Wild/Scenic Tributary or

0.90 Wildife Value

Latitude $\quad 3326.00$

Upstream/Downstream wild/Scenic Location

cultural value

Fish Presence Value

Geologic Value

Historic Value

other Value

Recreation value

Threatened/Endangered Fish

Threatened/Endangered Wildlife

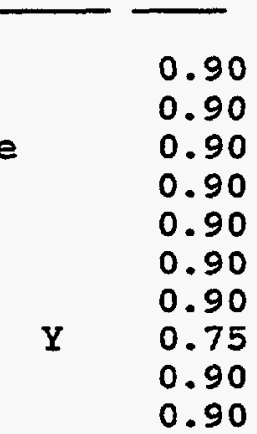

Scenic Value

0.90 Federal Land code 103

0.90 Federal Iand code 104

0.90 Federal Land Code 105

0.90 Federal Land Code 106

0.90 Federal Land Code 107

0.90 Federal Land code 108

0.90 Federal Land Code 198

0.90 
R E S O UR C E D A T A B A S E I I S T I N G

DATE: $08 / 26 / 97$

FERC

Number

Plant Name

11057 ROOSEVELT (RWCD)

County Name

MARICOPA

Class

M ROOSEVELT WTR CONS DISTRICT

Name Plate

Rating (KW)

PESF

PESF*KW

Annual Energy

3200.00

$0.90 \quad 2880.00$

ROOSEVELT CNL (SALT R)

River Basin

GIIA RIVER BASIN

PAGE NO: 5

State

Name

AZ

PESF Annual

\begin{tabular}{|c|c|c|c|c|}
\hline $\begin{array}{l}\text { Unit } \\
\text { Type }\end{array}$ & $\begin{array}{l}\text { Plant } \\
\text { Type }\end{array}$ & $\begin{array}{l}\text { Project } \\
\text { status }\end{array}$ & $\begin{array}{l}\text { Dam } \\
\text { status }\end{array}$ & $\begin{array}{lr}\text { Latitude } & 3328.00 \\
\text { Longitude } 11144.00\end{array}$ \\
\hline C & DIV & $x x$ & $\mathrm{U}$ & \\
\hline
\end{tabular}

Factor

Exist Prob

Factor

Wild/Scenic Protection

wild/Scenic Tributary or

0.90 Wildlife Value Energy Rating (MWh)

Upstream/Downstream wild/Scenic Location

Cultural value

Fish Presence Value

Geologic Value

Historic Value

other Value

Recreation Value

Scenic Value

Threatened/Endangered Fish

Threatened/Endangered wildlife

0.90 Federal Land Code 103

0.90 Federal Land Code 104

0.90 Federal Land Code 105

0.90 Federal Land Code 106

0.90 Federal Land Code 107

0.90 Federal Land Code 108

0.90 Federal Land Code 198

0.90
Exist Prob

25200

\begin{tabular}{ll}
\multicolumn{1}{c}{ Exist } & Prob \\
& \\
Fish & 0.90 \\
Wildlife & 0.90 \\
& 0.90 \\
& 0.90 \\
& 0.90 \\
& 0.90 \\
& 0.90 \\
& 0.90 \\
& 0.90 \\
& 0.90
\end{tabular}


$R E S O U R C E$

DATE: $08 / 26 / 97$
D A T A B A S E

I I S I N G
FERC

Number

11178
Plant Name

TUCSON

county Name

PIMA
PAGE NO: 6

state

Name

TUCSON WS PL (CAP AQUADUCT)

AZ
River Basin

GILA RIVER BASIN

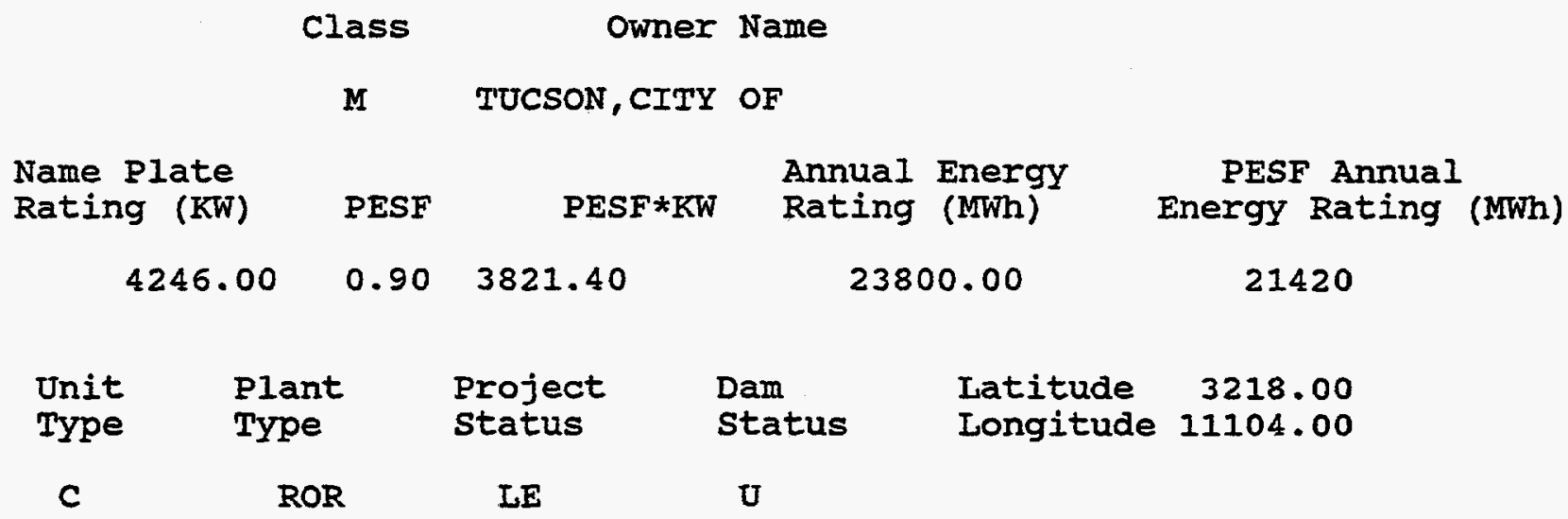

Factor

Wild/Scenic Protection

wild/Scenic Tributary or Upstream/Downstream wild/Scenic Location Cultural Value Fish Presence Value Geologic Value Historic Value other Value Recreation Value Scenic Value
Exist Prob

Factor

Exist Prob

0.90

Wildife Value Threatened/Endangered Fish Threatened/Endangered Wildlife

0.90 Federal Land Code 103

0.90 Federal Land Code 104

0.90 Federal Land Code 105

0.90 Federal Land Code 106

0.90 Federal Land Code 107

0.90 Federal Iand Code 108

0.90 Federal Land Code 198

0.90
0.90

0.90

0.90

0.90

0.90

0.90

0.90

0.90

0.90

0.90 
RES O URCE DA T A B A S E I I S T I N G

DATE: $08 / 26 / 97$

PAGE NO: 7

FERC

Number

Plant Name

Stream

State

AZ001

ARIZONA FALLS

ARIZONA CNI

AZ

county Name

MARICOPA
River Basin

GIIA RIVER BASIN

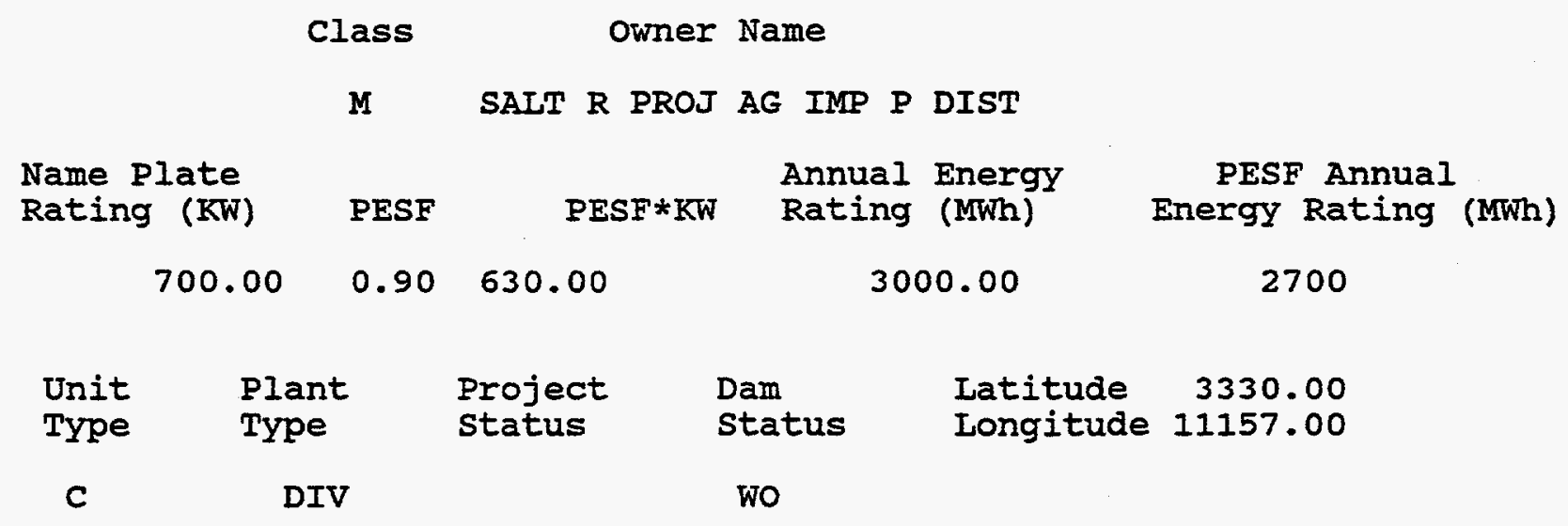

Factor

Wild/scenic Protection

wild/Scenic Tributary or Upstream/Downstream wild/Scenic Location Cultural Value Fish Presence Value Geologic Value Historic Value Other Value Recreation Value Scenic Value
Exist Prob

Factor

\subsection{Wildlife Value}

Threatened/Endangered Fish Threatened/Endangered Wildlife

0.90 Federal Land Code 103

0.90 Federal Land Code 104

0.90 Federal Land Code 105

0.90 Federal Land Code 106

0.90 Federal Land Code 107

0.90 Federal Land Code 108

0.90 Federal Land Code 198

0.90
Exist Prob

\begin{tabular}{|c|c|}
\hline $\mathbf{E}$ & Prob \\
\hline $\begin{array}{l}\text { Fish } \\
\text { Wildlife }\end{array}$ & $\begin{array}{l}0.90 \\
0.90 \\
0.90 \\
0.90 \\
0.90 \\
0.90 \\
0.90 \\
0.90 \\
0.90 \\
0.90\end{array}$ \\
\hline
\end{tabular}


R E S O U R C E

DATE: $08 / 26 / 97$
D A T A B A S E

PAGE NO: 8

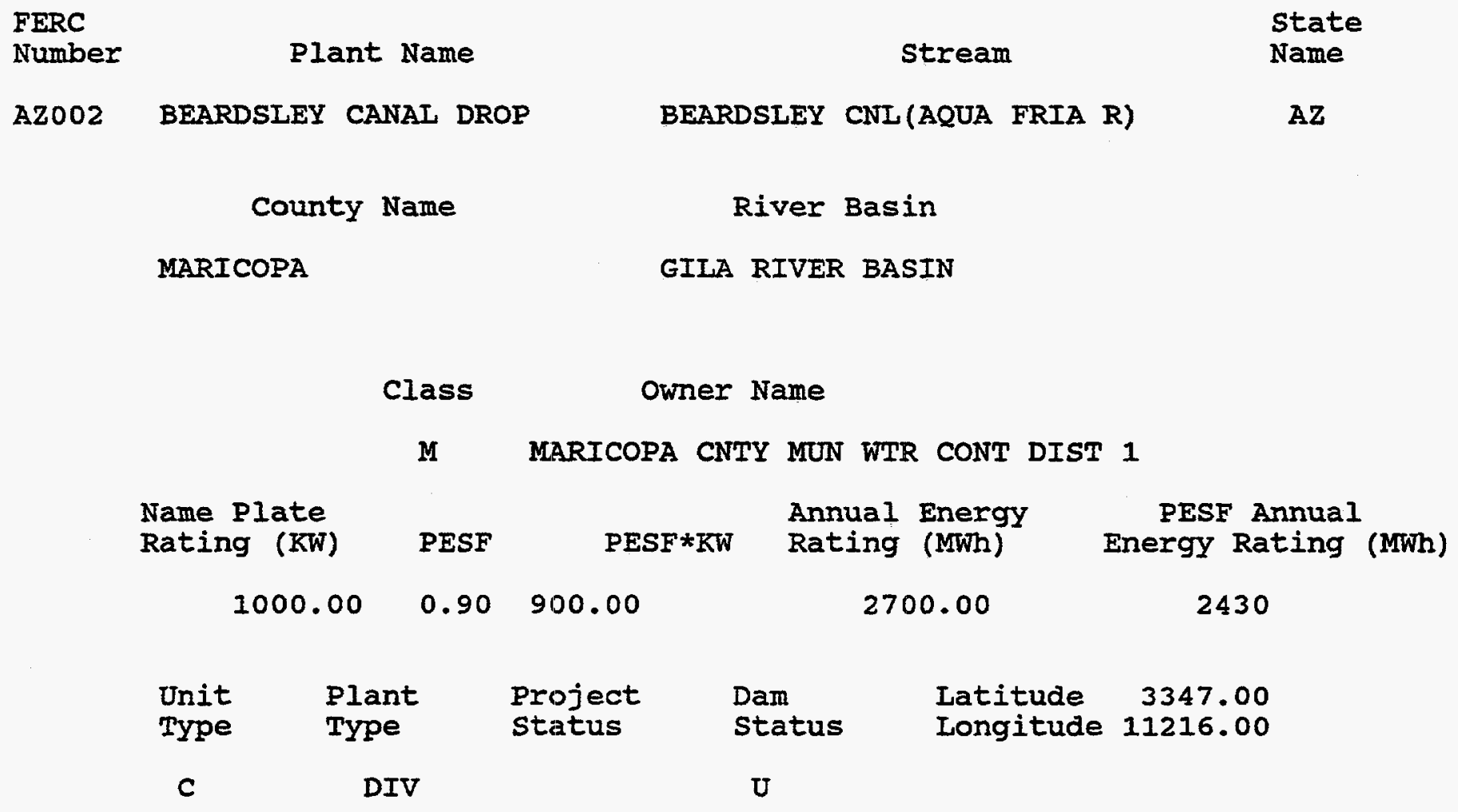

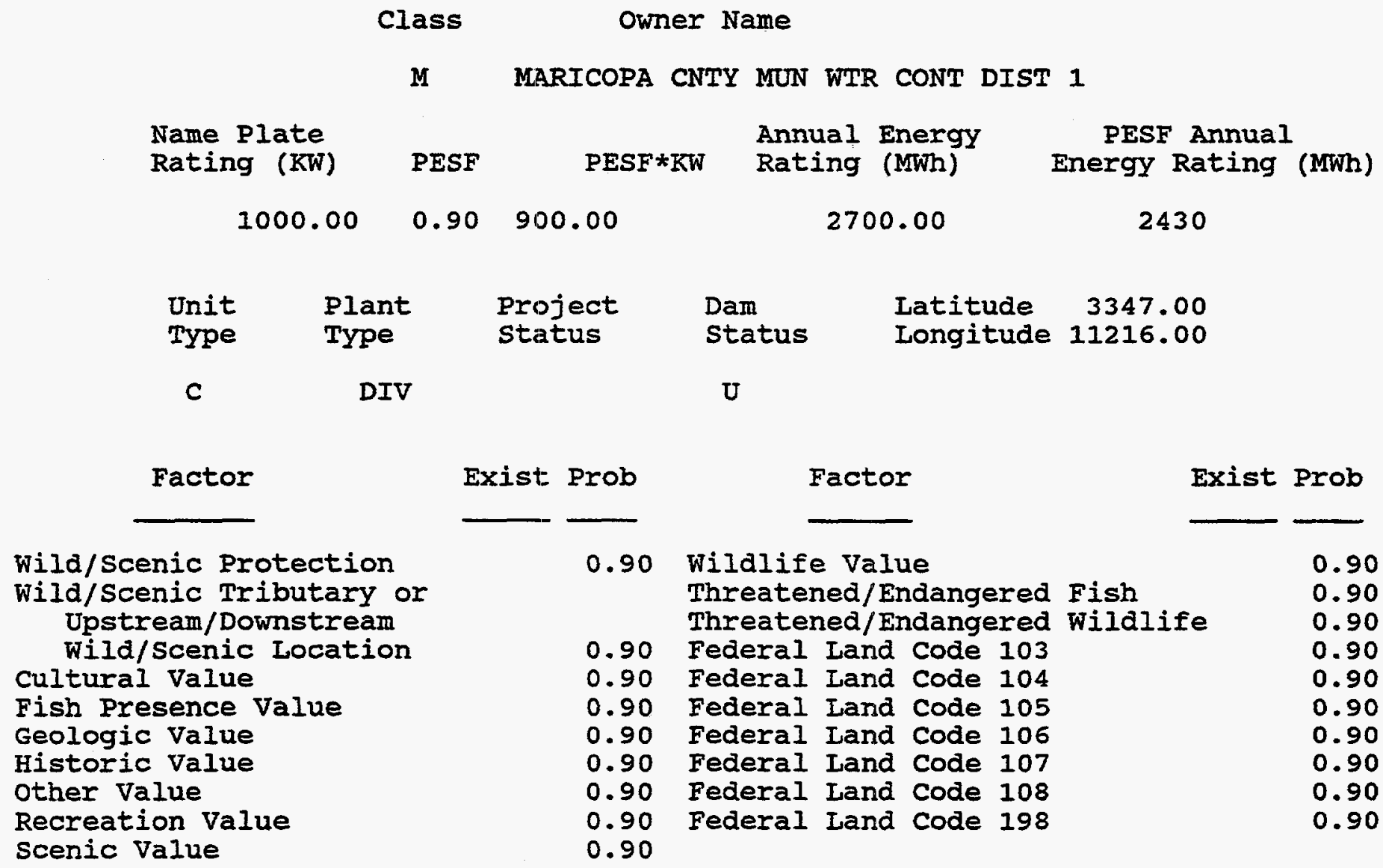


RESOURCE DATABASE I IS TING

DATE: $08 / 26 / 97$

PAGE NO: 9

FERC

Number

Plant Name

Stream

state

2003

CAP CANAI TURNOUT

CAP CNL(AQUA FRIA R)

Name

AZ003

county Name

River Basin

MARICOPA

GIIA RIVER BASIN

$\mathrm{AZ}$

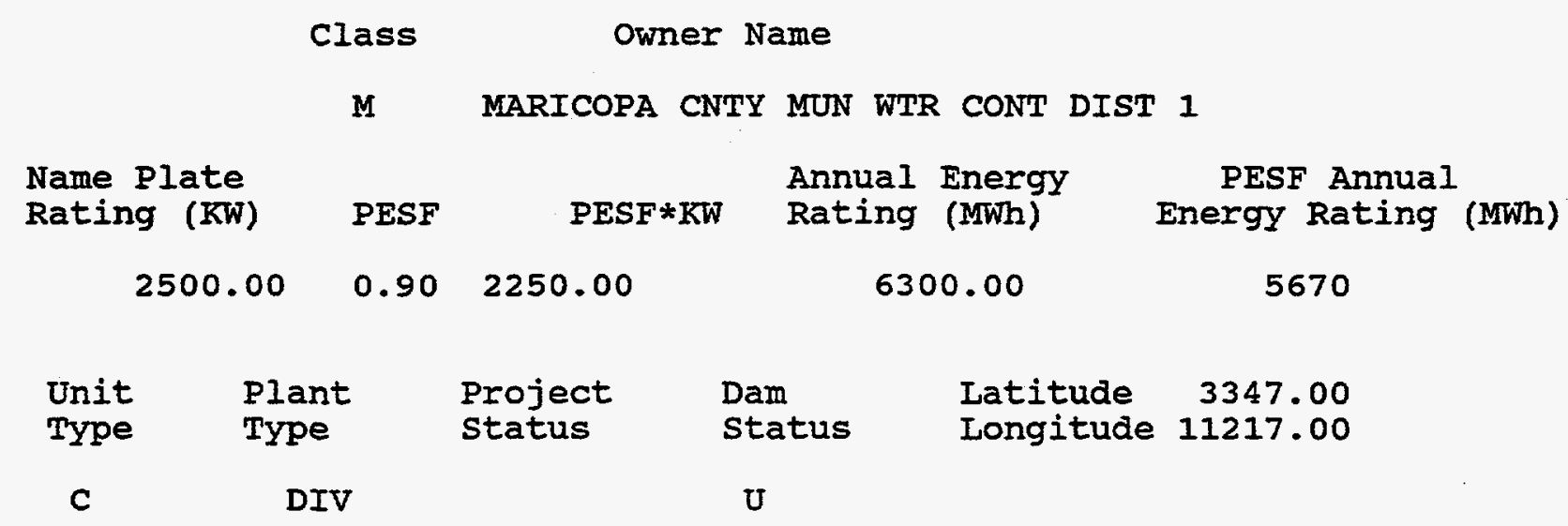

Factor

Exist Prob

Factor

Exist Prob

Wild/Scenic Protection

wild/Scenic Tributary or

0.90 Wildlife Value

Threatened/Endangered Fish

0.90

Upstream/Downstream

wild/Scenic Location

Threatened/Endangered Wildlife

0.90

0.90 Federal Land Code 103

0.90

Cultural Value

0.90 Federal Land code 104

0.90

0.90 Federal Iand Code 105

0.90

Fish Presence Value

0.90 Federal Land Code 106

0.90

Geologic Value

0.90 Federal Land Code 107

0.90

Historic Value

Other Value

Recreation value

0.90

Federal Iand Code 108

0.90

0.90

Federal Land Code 198

0.90

Scenic Value

0.90

0.90 
R E S O U R C E

DATE: $08 / 26 / 97$

FERC

Number

Plant Name

$\mathrm{AZ004}$

GLEASON FIAT

county Name

GILA

Class

Owner Name

$\mathbf{M}$

SALT R PROJ AG IMP P DIST

Name Plate

Rating (KW)

PESF

PESF*KW

Annual Energy

43500.00

0.10

4350.00

stream

River Basin

GILA RIVER BASIN
I I S I N G

PAGE NO: 10 state

Name

AZ

\begin{tabular}{|c|c|c|c|c|}
\hline $\begin{array}{l}\text { Unit } \\
\text { Type }\end{array}$ & $\begin{array}{l}\text { Plant } \\
\text { Type }\end{array}$ & $\begin{array}{l}\text { Project } \\
\text { status }\end{array}$ & $\begin{array}{l}\text { Dam } \\
\text { Status }\end{array}$ & $\begin{array}{lr}\text { Latitude } 3345.00 \\
\text { Longitude } 11042.00\end{array}$ \\
\hline C & DIV & & U & \\
\hline
\end{tabular}

Factor

Exist Prob

Factor

Exist Prob

wild/Scenic Protection

wild/Scenic Tributary or Upstream/Downstream wild/Scenic Iocation

$\mathbf{Y}$

$$
0.10
$$

wilalife Value

Threatened/Endangered Fish

Threatened/Endangered Wildlife

0.90

0.50

Federal Land Code 103

Federal Land Code 104

Federal Land Code 105

0.25

0.50

Federal Iand Code 106

0.90 Federal Land Code 107

Historic value

Other Value

Recreation Value

Scenic Value

$\begin{array}{lll}Y & 0.50 & \text { Federal Land Code } 108 \\ Y & 0.25 & \text { Federal Land Code } 198\end{array}$

$\begin{array}{lll}Y & 0.50 & \text { Federal Land Code } 108 \\ Y & 0.25 & \text { Federal Land Code } 198\end{array}$

$\begin{array}{llll}\mathrm{Y} & 0.50 & \text { Federal Land Code } 108 \\ \mathrm{Y} & 0.25 & \text { Federal Land Code } 198\end{array}$

$\mathrm{Y} \quad 0.50$
PESF Annual Energy Rating (MWh)

0.25

0.50

0.50

0.90

0.50

0.90

0.90

0.90

0.90

0.90 
DATE : $08 / 26 / 97$

FERC

Number

AZ005

GLEN CANYON

County Name

COCONINO

Class

F BUREAU OF RECLAMATION

Name Plate Rating ( $\mathrm{KW})$

71816.00

PESF

PESF*KW

Annual Energy

Rating (MWh)

$0.50 \quad 35908.00$

367784.00

Unit Plant

Type

Type

C

STG

Factor

Exist Prob

Wild/Scenic Protection

wild/Scenic Tributary or

Upstream/Downstream

wild/Scenic Location

Cultural value

Fish Presence Value

Geologic Value

Historic Value

other Value

Recreation value

Scenic Value

River Basin

COLORADO MAIN STREAM
PAGE NO: 11

state

Name

AZ
PESF Annual Energy Rating (MWh)

183892
Dam

Project status

FA

Factor

Latitude $\quad 3656.00$

Status

W

\begin{tabular}{|c|c|c|c|c|c|c|c|c|}
\hline & 0.90 & $\begin{array}{l}\text { Wildilf } \\
\text { Threater } \\
\text { Threater }\end{array}$ & $\begin{array}{l}\text { e Val } \\
\text { ned/E } \\
\text { ned/E }\end{array}$ & $\begin{array}{l}\text { ue } \\
\text { ndang } \\
\text { ndang }\end{array}$ & $\begin{array}{l}\text { ered } \\
\text { ered }\end{array}$ & $\begin{array}{l}\text { Fish } \\
\text { Wildilf }\end{array}$ & $\begin{array}{l}Y \\
Y\end{array}$ & $\begin{array}{l}0.75 \\
0.75 \\
0.90\end{array}$ \\
\hline & 0.90 & Federal & Land & Code & 103 & & & $\begin{array}{l}0.90 \\
0.75\end{array}$ \\
\hline$Y$ & 0.75 & Federal & Land & Code & 104 & & $Y$ & 0.7 \\
\hline$Y$ & 0.75 & Federal & Land & Code & 105 & & $Y$ & 0.5 \\
\hline$Y$ & 0.90 & Federal & Land & Code & 106 & & & 0.90 \\
\hline$Y$ & 0.75 & Federal & Land & Code & 107 & & & 0.90 \\
\hline$Y$ & 0.75 & Federal & Land & Code & 108 & & & 0.90 \\
\hline$Y$ & 0.75 & Federal & Land & code & 198 & & & \\
\hline
\end{tabular}


R E S O U R E

DATE: $08 / 26 / 97$
D A T A B A S E

L I S T I N G

PAGE NO: 12

FERC

Number

Plant Name

stream

State

Name

$\begin{array}{rr}\text { AZ006 HEADGATE ROCK } & \text { COLORADO R } \\ \text { County Name } & \text { River Basin } \\ \text { YUMA } & \text { GIIA RIVER BASIN }\end{array}$

Class Owner Name

F BUREAU OF INDIAN AFFAIRS

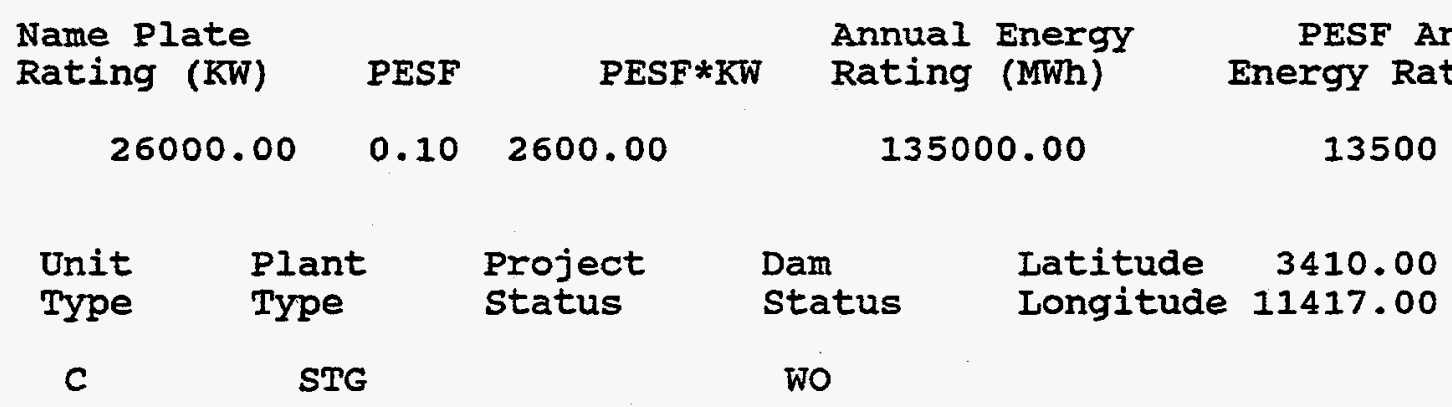

Factor

Exist Prob

Factor

Exist Prob

Wild/Scenic Protection

wild/scenic Tributary or Upstream/Downstream wild/Scenic Location Cultural Value Fish Presence Value Geologic Value Historic Value other Value Recreation Value Scenic value

Exist Pro

$$
0.90
$$

Wildlife Value Threatened/Endangered Fish Threatened/Endangered Wildlife

0.90 Federal Land code 103

0.90 Federal Iand code 104

0.90 Federal Iand code 105

0.90 Federal Iand code 106

0.90 Federal Land code 107

Y 0.75 Federal Land code 108

Y 0.75 Federal Land code 198

$\begin{array}{ll}Y & 0.75 \\ Y & 0.90\end{array}$
AZ 
R E S O U R E D A T A B A S E E I S T I N G

DATE: $08 / 26 / 97$

PAGE NO: 13

FERC

Number

Plant Name

stream

State

AZ007 HOOVER

COLORADO R

Name

county Name

River Basin

COCHISE

GIIA RIVER BASIN

Class Owner Name

F BUREAU OF RECLAMATION

Name Plate

Rating ( $\mathrm{KW}$ )

PESF

Annual Energy

135000.00

$0.90 \quad 121500.00$

Rating (MWh)

PESF Annual

Energy Rating (MWh)

135000.00

$\begin{array}{cc}\text { Unit } & \text { Plant } \\ \text { Type } & \text { Type } \\ \text { C } & \text { STG }\end{array}$

Project

Status

$\begin{array}{llr}\text { Dam } & \text { Latitude } & 3601.00 \\ \text { Status } & \text { Longitude } & 11444.00\end{array}$

FA

W

Factor

Exist Prob

Factor

Exist Prob

Wild/Scenic Protection

wild/Scenic Tributary or

0.90 Wildlife Value

Threatened/Endangered Fish

270589.50

AZ

Upstream/Downstream wild/Scenic Location Threatened/Endangered Wilalife

0.90 Federal Land code 103

0.90 Federal Land code 104

0.90 Federal Land code 105

0.90 Federal Land code 106

0.90 Federal Land code 107

0.90 Federal Iand code 108

0.90 Federal Land Code 198

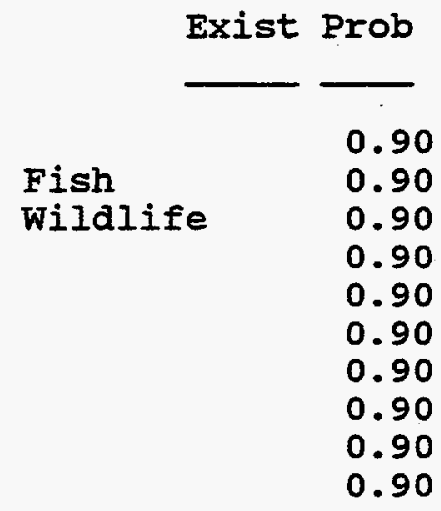

Recreation value

0.90

0.90 


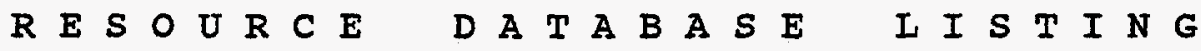

DATE: $08 / 26 / 97$

PAGE NO: 14

FERC

Number

Plant Name

stream

State

AZ008

HUALAPAI

COLORADO $\mathrm{R}$

Name

AZ

county Name

MOHAVE
River Basin

COLORADO MAIN STREAM

class Owner Name

NHPS/COE

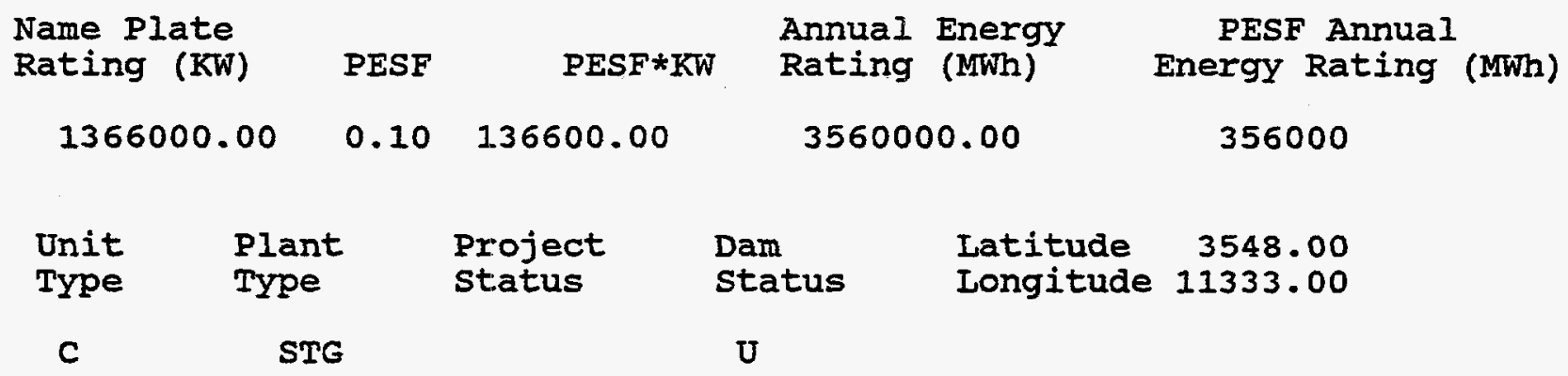

Factor

Exist Prob

Factor

Exist Prob

Wild/Scenic Protection

wild/Scenic Tributary or

Upstream/ Downstream

wild/Scenic Location

Cultural Value

Fish Presence Value

Geologic Value

Historic Value

other Value

Recreation value

Scenic Value

Exist Prob

Y 0.10 Wildlife Value

Threatened/Endangered Fish

Y 0.25

Threatened/Endangered Wildlife

0.90 Federal Land Code 103

$Y \quad 0.50 \quad$ Federal Iand Code 104

$Y \quad 0.25$ Federal Iand Code 105

$Y \quad 0.50 \quad$ Federal Land Code 106

$Y \quad 0.50 \quad$ Federal Land Code 107

Y $\quad 0.50$ Federal Land Code 108

Y 0.25 Federal Land Code 198

0.90

$Y \quad 0.50$

0.90

0.10

0.90

0.90

0.90

0.90

0.90

0.90 
R E S O UR C E D A T A B A S E I IS T ING

DATE: $08 / 26 / 97$

PAGE NO: 15

FERC

Number

Plant Name

Stream

State

Name

AZ009 KNOB

SALT R

AZ

County Name

River Basin

GILA

GIIA RIVER BASIN

Class Owner Name

M SAIT R PROJ AG IMP P DIST

$\begin{array}{rlcc}\begin{array}{l}\text { Name Plate } \\ \text { Rating (KW) }\end{array} & \text { PESF } & \text { PESF*KW } & \begin{array}{c}\text { Annual Energy } \\ \text { Rating }\end{array} \\ & & & \end{array}$

PESF Annual

Energy Rating (MWh)

28500.00

$0.10 \quad 2850.00$

$\begin{array}{llr}\text { Dam } & \text { Latitude } & 3347.00 \\ \text { Status } & \text { Longitude } & 11024.00\end{array}$

$\begin{array}{lll}\text { Unit } & \text { Plant } & \text { Project } \\ \text { Type } & \text { Type } & \text { Status }\end{array}$

C

STE

U

Factor

Exist Prob

\subsection{0}

wild/Scenic Tributary or Upstream/Downstream wild/Scenic Location Cultural Value

Fish Presence Value

Geologic Value

Historic Value

other Value

Recreation value

Scenic Value
Factor

Wildlife Value Threatened/Endangered Fish Threatened/Endangered Wildiife $Y$

0.90 Federal Land Code 103

$Y \quad 0.50$ Federal Land Code 104

$Y \quad 0.25$ Federal Land Code 105

Y $\quad 0.50$ Federal Land Code 106

0.90 Federal Land Code 107

$Y \quad 0.50$ Federal Land Code 108

$\mathrm{Y} \quad 0.25$ Federal Land Code 198
13600 
R E S O U C E

DATE: $08 / 26 / 97$
D A T A A S E

FERC

Number

AZ010

IIVINGSTONE

county Name

GILA
Plant Name
PINAL CR, SAIT R

River Basin

GIIA RIVER BASIN

PAGE NO: 16

I IS T I N G

State

Name

AZ

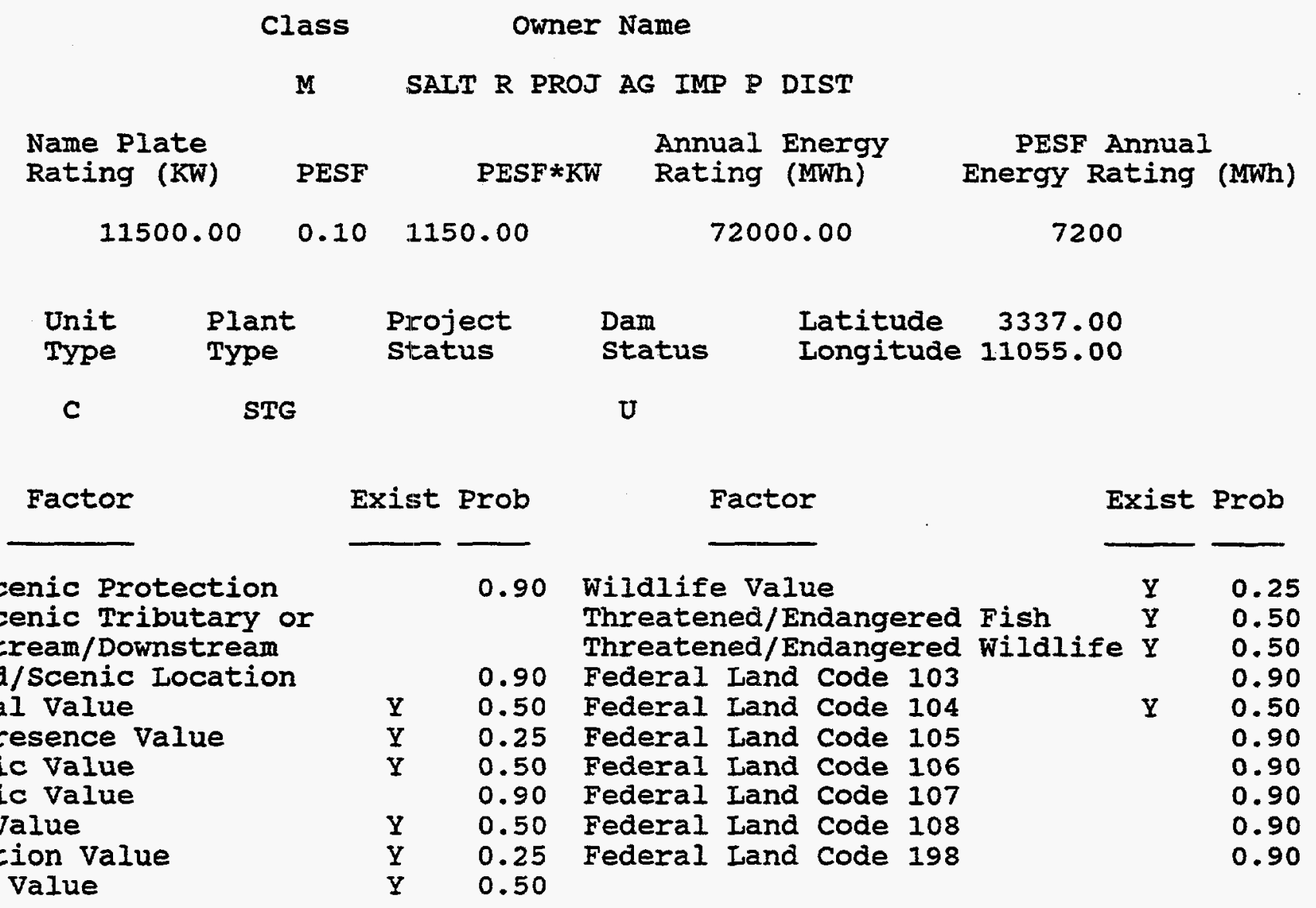

wild/Scenic Protection

Wild/Scenic Tributary or Upstream/Downstream wild/Scenic Location Cultural value Fish Presence Value

Geologic Value

Historic Value

other Value

Recreation Value

Scenic Value

Exist Prob

0.90

Threatened/Endan

Federal Land Code 103

Federal Land Code 104

code 105

Federal Land Code 108

Federal Land Code 198 
RESOURCE DATABASE I IS T ING

DATE: $08 / 26 / 97$

PAGE NO: 17

FERC

Number

Plant Name

stream

State

Name

AZ011 MULE HOOF

SALT $R$

AZ

County Name

River Basin

GIIA

GILA RIVER BASIN

Class Owner Name

M SAIT R PROJ AG IMP P DIST

Name Plate

PESF $\quad$ PESF*KW $\begin{aligned} & \text { Annual Energy } \\ & \text { Rating (MWh) }\end{aligned}$

PESF Annual

Rating ( $\mathrm{KW}$ )

$0.10 \quad 4350.00$

280000.00

Energy Rating (MWh)

$$
43500.00
$$

$\begin{array}{cc}\text { Unit } & \text { Plant } \\ \text { Type } & \text { Type } \\ \text { C } & \text { DIV }\end{array}$

Project

status

Factor

Exist Prob

Factor

Latitude $\quad 3348.00$

Status

Longitude 11031.00

U
wild/Scenic Protection

wild/Scenic Tributary or Upstream/Downstream wild/Scenic Location Cultural Value Fish Presence Value Geologic Value Historic Value other Value Recreation value Scenic Value

\section{Exist Prob}

0.90

Wildifie Value Threatened/Endangered Fish Threatened/Endangered
Federal Land Code 103

$\begin{array}{llll} & 0.90 & \text { Federal Land Code } 103 \\ \mathrm{Y} & 0.50 & \text { Federal Land Code } 104 \\ \mathrm{Y} & 0.25 & \text { Federal Land Code } 105\end{array}$

$\mathrm{Y} \quad 0.50$

Federal Land Code 106

0.90

Federal Land Code 107

0.50

0.25

0.50
28000 
R E S O U R C E

DATE: $08 / 26 / 97$
D A T A B A S E

FERC

Number

plant Name

AZ012 ORME 3

County Name

GILA

GIIA RIVER BASIN
L I S T I N G

PAGE NO: 18

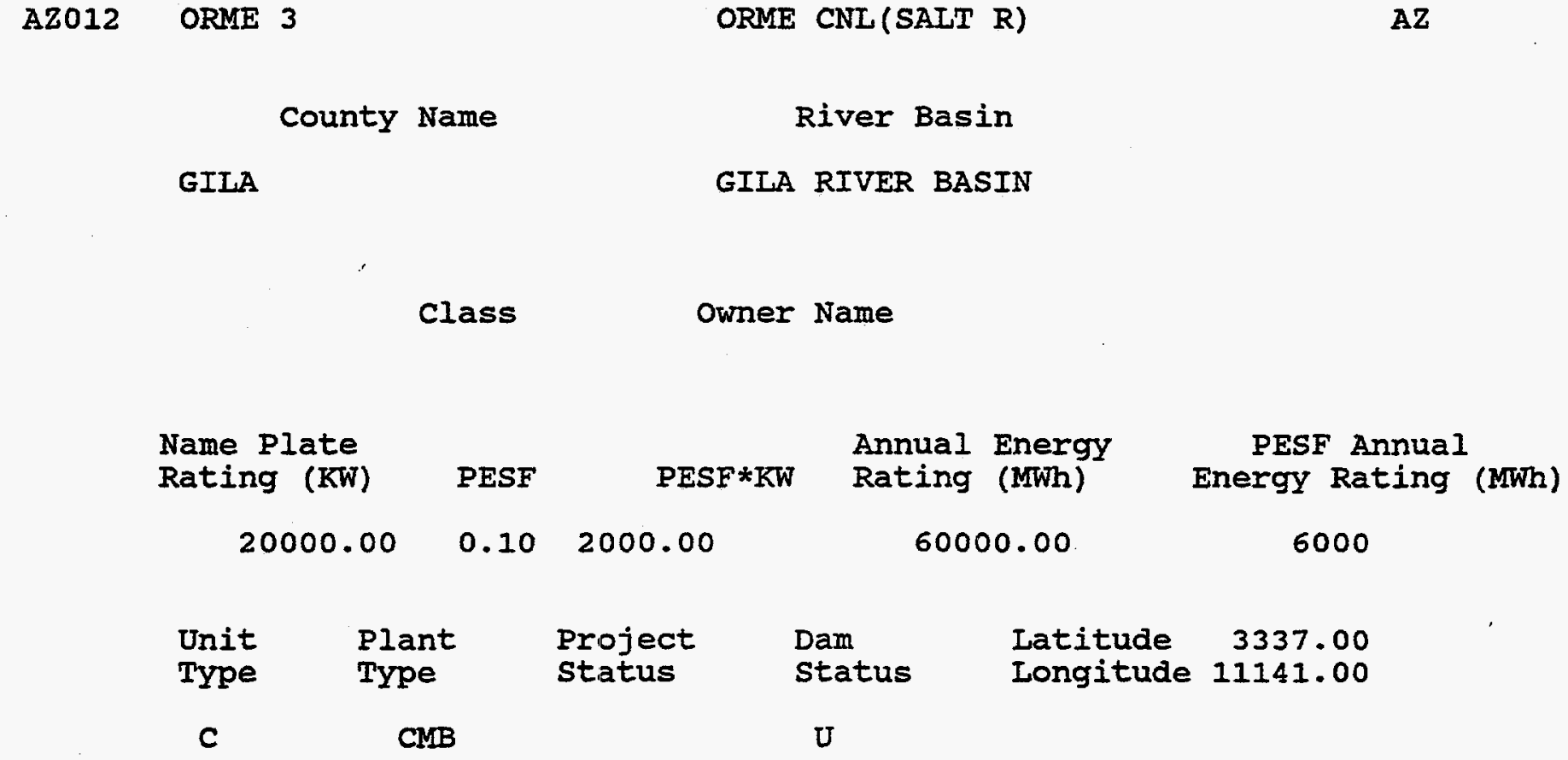

Factor

wild/Scenic Protection

wild/Scenic Tributary or Upstream/Downstream wild/Scenic Location Cultural value Fish Presence Value Geologic Value Historic Value other Value Recreation Value Scenic Value
Exist Prob

Factor

Exist Prob

$\begin{array}{ll} & \\ & 0.90 \\ & \\ & 0.90 \\ Y & 0.50 \\ Y & 0.25 \\ Y & 0.50 \\ Y & 0.90 \\ Y & 0.50 \\ Y & 0.25 \\ & 0.50\end{array}$

\section{Wildlife Value}

Threatened/Endangered Fish Threatened/Endangered Wildlife $Y$ Federal Land code 103 Federal Land Code 104 Federal Land code 105 Federal Land Code 106 Federal Land Code 107 Federal Iand Code 108 Federal Iand code 198 state Name

AZ 
DATE: $08 / 26 / 97$

PAGE NO: 19

FERC

Number

Plant Name

Stream

State

Name

A2013 WADDELI

AQUA FRIA $R$

AZ

County Name

River Basin

MARICOPA

GIIA RIVER BASIN

Class Owner Name

M MARICOPA CNTY MUN WTR CONT DIST 1

Name Plate

Annual Energy

Rating (KW)

PESF

PESF*IW

Rating (MWh)

PESF Annual

1500.00

$0.90 \quad 1350.00$

3300.00

2970

$\begin{array}{lllll}\text { Unit } & \text { Plant } & \text { Project } & \text { Dam } & \text { Latitude } 3351.00 \\ \text { Type } & \text { Type } & \text { Status } & \text { Status } & \text { Longitude 11216.00 }\end{array}$

C

STG

U

Factor

Exist Prob

Factor

Exist Prob

ild/Scenic Protection Wild/Scenic Tributary or

0.90 Wildlife Value Threatened/Endangered Fish

0.90

Upstream/Downstream Threatened/Endangered Wildlife

0.90

wild/Scenic Location

0.90 Federal Land code 103

0.90 Federal Land code 104

0.90

Cultural value

0.90 Federal Land Code 105

0.90

Fish Presence Value

0.90 Federal Land Code 106

0.90

Geologic Value

0.90

Federal Land Code 107

0.90

Historic Value

0.90

Federal Land code 108

0.90

other Value

0.90

Federal Land Code 198

0.90

Recreation Value

0.90

.90

Scenic Value

0.90 
R E S O U C E

DATE: $08 / 26 / 97$
D A T A B A S

FERC

Number

Plant Name

A2014

WALNUT

CANYON

County Name

GILA

class

Owner Name

M

SALT R PROJ AG IMP P DIST

Name Plate

PESF

$\mathrm{PESF} * \mathrm{KW}$ Rating

Rating (KW)

$0.10 \quad 2520.00$

25200.00

Unit
Type

Plant

Type

Project St:atus

C

STG

Factor

Exist Prob

Factor

Latitude

status

Longitude 11027.00

U
State

Name

AZ

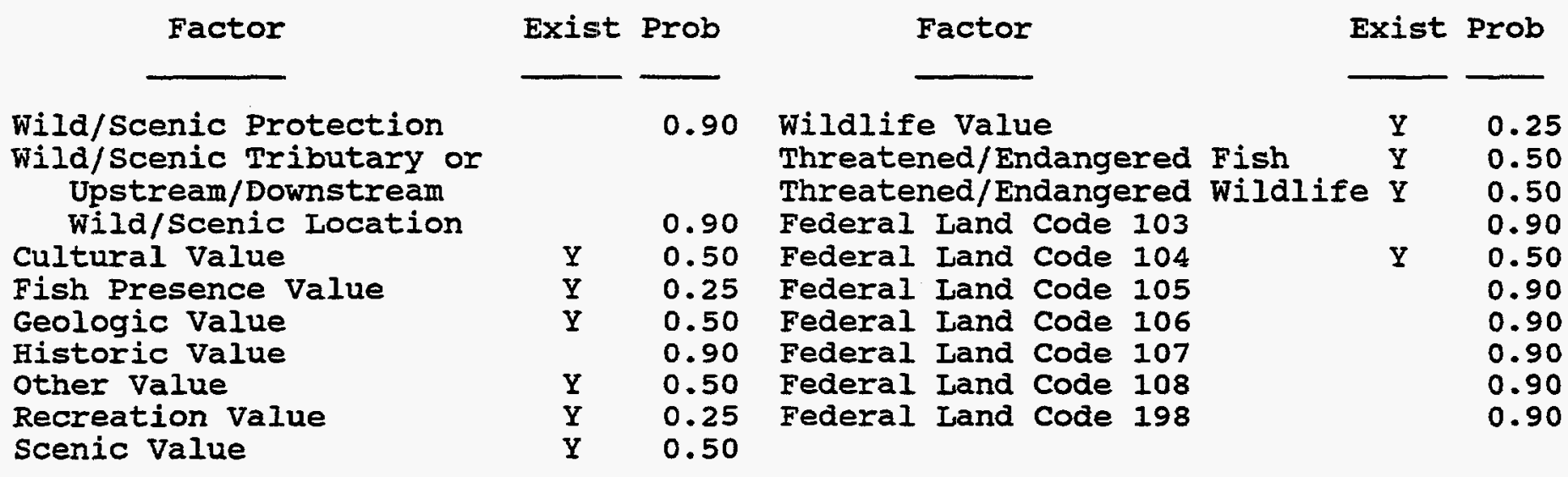


R E S U U C E D A T A B A S E I I S T I N G

DATE: $08 / 26 / 97$

PAGE NO: 21

FERC

Number

Plant Name

Stream

State

AZ015 YUMA MAIN CANAI

YUMA MN CNL (GILA R)

Name

AZ

County Name

River Basin

YUMA

GILA RIVER BASIN

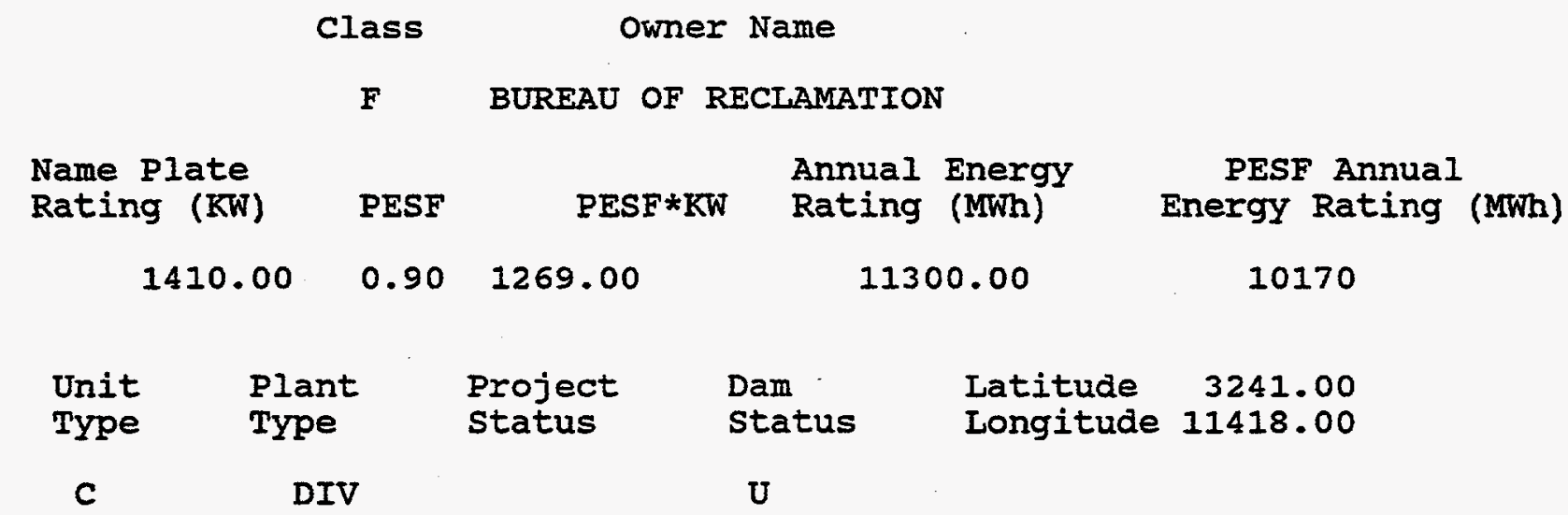

Factor

Exist Prob

Factor

Exist Prob

Wild/Scenic Protection

Wild/Scenic Tributary or

0.90 Wildlife Value

Threatened/Endangered Fish

Upstream/Downstream Threatened/Endangered Wildlife wild/Scenic Location

0.90 Federal Iand Code 103

0.90 Federal Iand Code 104

0.90 Federal Land Code 105

0.90 Federal Land Code 106

0.90 Federal Land Code 107

0.90 Federal Land Code 108

0.90 Federal Land Code 198

0.90

0.90

0.90

0.90

Cultural Value

Geologic Value

0.90

0.90

0.90

0.90

0.90

0.90

Other Value

0.90

Scenic Value 